\title{
First beam test of UT sensors with the SALT 3.0 readout ASIC
}

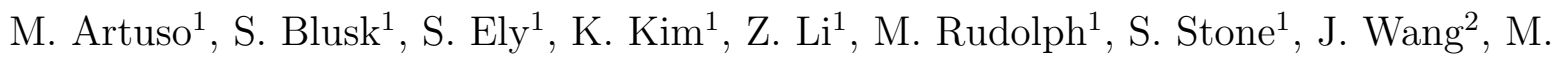 \\ Wilkinson $^{1}$ \\ ${ }^{1}$ Syracuse University, Syracuse, NY 13244, USA \\ ${ }^{2}$ Institute of High Energy Physics, Beijing, China
}

\begin{abstract}
This note presents the results of a beam test conducted at the Fermilab test beam facility in March 2019. The beam test represents the first such test of the UT readout ASIC, SALT 3.0, in a beam with production UT sensors. Two sensors were tested, one an unirradiated p-in-n sensor, and the second a n-in-p sensor irradiated to twice the maximum expected fluence. The beam test demonstrates that the SALT 3.0 ASIC meets our specifications, and can be used for the production. Due to specific issues with the data acquisition system that will not be present in the system in LHCb, a higher threshold was used in the beam test, corresponding to about $6 \sigma$ on the noise. With this higher threshold, the signal-to-noise ratio $(\mathrm{S} / \mathrm{N})$ and efficiency are measured. For the unirradiated sensor, a $\mathrm{S} / \mathrm{N}$ of about 12 is achieved, with a single-hit efficiency of at least $99.5 \%$. For the irradiated sensor, a $\mathrm{S} / \mathrm{N}$ of about 11 is obtained, with a single-hit efficiency of about $94 \%$. In the final system in LHCb with a proper threshold of about 3-4 $\sigma$ of the noise, we expect to achieve a single-hit efficiency of at least $99.5 \%$.
\end{abstract}

This manuscript has been authored by Fermi Research Alliance, LLC under Contract No. DE-AC02-07CH11359 with the U.S. Department of Energy, Office of Science, Office of High Energy Physics.

(C) 2019 CERN for the benefit of the LHCb collaboration. CC-BY-4.0 licence. 



\section{Introduction}

2 The Upstream Tracker (UT) detector is a key part of the LHCb Upgrade, replacing the 3 TT stations. A detailed description of the UT is given in the Technical Design Report [1]. 4 The UT detector consists of four large-area silicon planes, each about $1.53 \mathrm{~m}$ in width 5 and $1.34 \mathrm{~m}$ in height. Each plane is composed of $1.5 \mathrm{~m}$ long staves that are tiled mostly 6 with $\sim 10 \mathrm{~cm} \mathrm{x} 10 \mathrm{~cm}$ silicon wafers. Consecutive wafers are mounted on opposite sides 7 of the stave to ensure no gaps along the height, and adjacent staves are also overlapped 8 to ensure no gaps in the horizontal direction. A cartoon of the UT detector is shown in 9 Fig. 1 .

The majority of the $10 \mathrm{~cm}$ x $10 \mathrm{~cm}$ in silicon sensors have strip pitch of $187.5 \mu \mathrm{m}$ [ green squares in Fig. 1], and are referred to as Type A sensors. The yellow squares are referred to as Type B, and are also $10 \mathrm{~cm} \times 10 \mathrm{~cm}$ in size, but represent sensors with approximately half the pitch $(93.5 \mu \mathrm{m})$ to cope with higher occupancy. In the innermost region are sensor types $\mathrm{C}$ and $\mathrm{D}$, which are $10 \mathrm{~cm} \times 5 \mathrm{~cm}$ in size, and also have the finer pitch. The type $\mathrm{D}$ sensors differ from the type $\mathrm{C}$ in that they have a $1 / 4$-circle cutout in order to bring the active detector as close to the beampipe as possible.

The Type A are all p-in-n type sensors, whereas types B, C and D are n-in-p, which have better performance under high irradiation than the p-in-n. The types A and B have nominal thicknesses of $320 \mu \mathrm{m}$, and the types $\mathrm{C}$ and D are nominally $250 \mu \mathrm{m}$ thick.

The readout chip for the UT is called the SALT (Silicon ASIC for LHCb Tracking) 2].

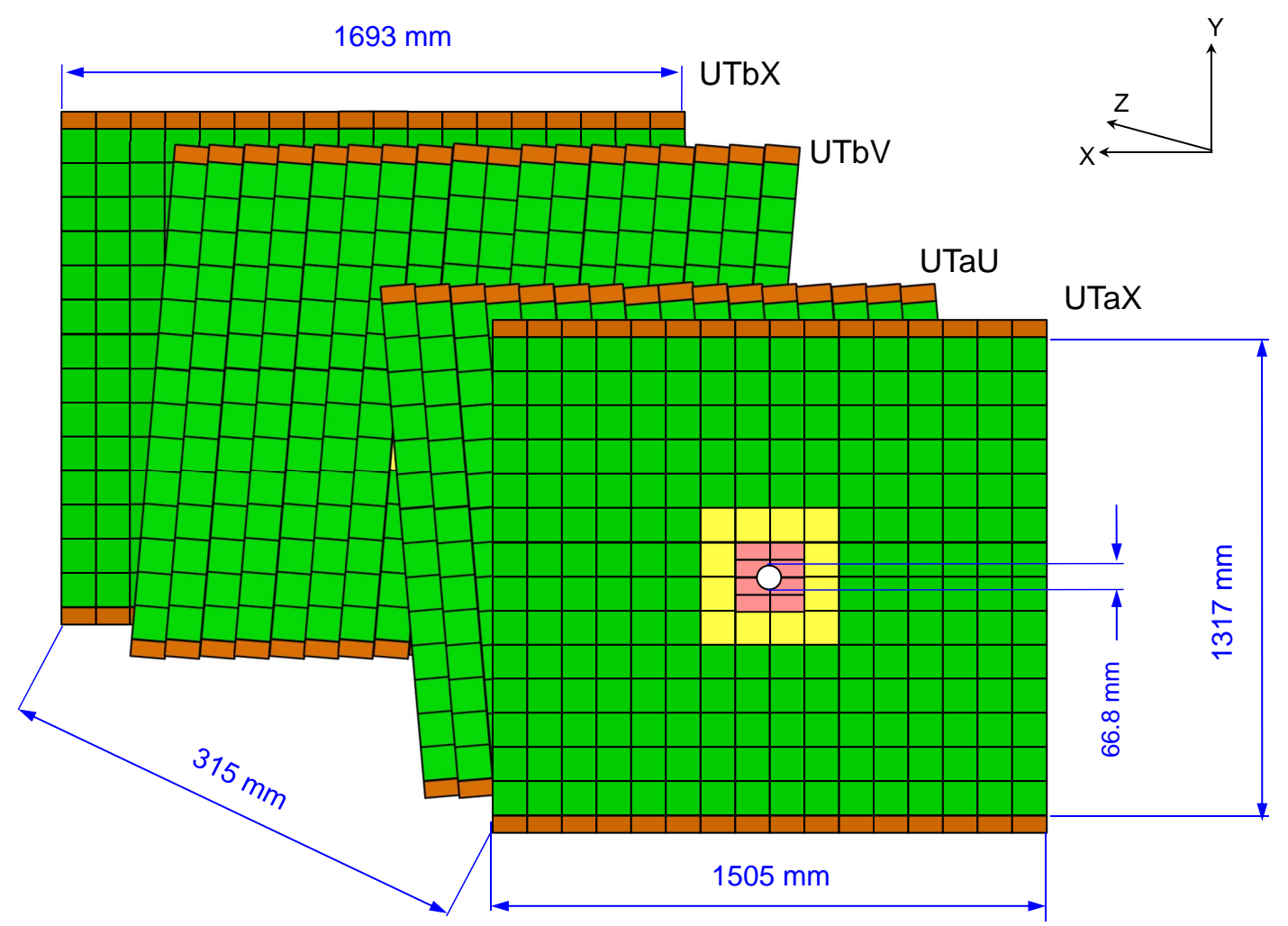

Figure 1: Cartoon showing the layout and dimensions of the four UT planes. 
It consists of 128 parallel channels each with a charge-sensitive pre-amplifiers, a shaper circuit, and a 6-bit SAR $\mathrm{ADC}$. The ADC provides a signed value, so one of the six bits is reserved for the sign of the charge. The SALT ASIC ${ }^{2}$ has gone through several versions, starting with 8-channel prototypes, up to full size 128-channel ASICs. The most recent version of the ASIC, SALT 3.0 [3], incorporates several necessary improvements to earlier versions. Initial bench tests showed that the revisions in SALT 3.0 addressed the most serious concerns with the earlier versions.

This note follows a number of previous beam tests performed on prototype UT sensors that were read out using Beetle [4] chips. Those studies are documented in Refs. [5, 6]. In this most recent beam test described here, two sensors were tested. One sensor was an unirradiated Type A sensor, and the second was a Type B sensor, uniformly irradiated at the CERN SPS to a fluence of $6.2 \times 10^{13} n_{e q} / \mathrm{cm}^{2}$. This is about twice the maximum expected fluence anticipated for Type B sensors in the UT. This work presents the results of the first beam test performed using the new SALT 3.0 readout chips with production UT sensors.

\section{Description of setup}

The beam test took place in March 2019 at the Fermilab Test beam facility (FTBF) [7]. The beam consists of $120 \mathrm{GeV}$ protons, and is bunched according to the $53 \mathrm{MHz}$ bunch structure of the Tevatron. Every minute, there is a 4.2 second spill, and within that spill there are $(4.2 \mathrm{~s})\left(53 \times 10^{6}\right) \simeq 2 \times 10^{8} \mathrm{RF}$ buckets. Typically, FTBF operates with the number of beam particles in the $2-5 \times 10^{4} /$ spill range, so that the intensity is relatively low, and the probability of multiple beam particles in a single RF bucket, or even consecutive RF buckets, is very low.

Beam particles were tracked with the FTBF telescope, which is composed of 7 pairs of silicon-strip detectors, each pair providing measurements in the horizontal $(x)$ and vertical (y) directions. The telescope planes have their own custom data acquisition (DAQ) system that is independent of the UT DAQ. A photo of the setup is shown in Fig. 2. Overall, a precision of about $10 \mu \mathrm{m}$ at the position of the UT detector under test (DUT) is expected.

The UT sensor is mounted in an aluminum box that provides for a dark environment, and also acts as a Faraday cage. The sensor is attached to a supporting board and the silicon strips are wirebonded to the SALT 3.0 ASICs, which are mounted on a hybrid. A GBT daughterboard packages and ships the data from all four ASICs over optical fibers to the MiniDAQ1 [8] DAQ system. A photo of the Type A sensor module is shown in Fig. 4 along with labels for each of the components shown.

The leakage current and capacitance of the two sensors to be tested in the beam test were measured prior to the beam test, and the results are shown in Fig. 3. Based upon the $C V$ curves, we expect the Type A to be fully depleted at approximately $150 \mathrm{~V}$. For the Type B, it should be at least $300 \mathrm{~V}$.

The UT system is operated in zero-suppressed (ZS) mode, where only UT channels above a prescribed threshold are read out. Reading out in non-zero suppressed mode is

\footnotetext{
${ }^{1} \mathrm{SAR}$ is short for Successive approximation register, and SAR ADCs allow for a fast, low power solution with a small footprint.

${ }^{2}$ ASIC is an acronym for Application Specific Integrated Circuit.
} 
only used for calibration purposes, and is not an option for the test beam. The SALT 3.0 ASIC allows for pedestal subtraction as well as functionality to perform dynamic common-mode noise subtraction (CMNS) on the data, prior to the application of the threshold. The CMNS feature largely removes the effects of noise sources that cause all channels to coherently shift their output levels up or down, i.e., common to all channels. Lastly, the SALT 3.0 has a TrimDAC, which allows one to adjust the zero of each channel's output. In the test beam, the TrimDAC of each channel was set to give an average ADC value of 1 , so that relative to this value, the $\mathrm{ADC}$ range is $-32 \leq \mathrm{ADC}<31$.

Hits over threshold from the SALT 3.0 are read out via the MiniDAQ1 data acquisition system [8] developed at CERN. The MiniDAQ1 groups the data into so-called multi-event packets (MEPs) [9], which are then sent to the host PC over ethernet. One of the issues with the MiniDAQ1 system was the loss of MEP data at the level of about $0.5 \%$, which needs to be accounted for when measuring the efficiency of the detector under test.

Figure 5 compares the total and common-mode subtracted noise (left) at the FTBF and (right) after the testbeam at Syracuse University for the Type A sensor. It shows the high level of total noise at the test beam of about 4 ADC counts. After common mode subtraction, the noise is about 0.95 ADC counts, on average. At SU, the total noise is only about $1.5 \mathrm{ADC}$ counts and the common mode subtracted noise is about $1 \mathrm{ADC}$. The Type B sensor gives similar results.

Custom software was developed to decode the UT MEP data and output the hit data for further offline analysis. Unlike in the case for non-zero suppressed data, where all channels are read out, ZS data requires a careful synchronization to ensure that UT hit data are assigned to the correct event. Here, an event can be considered to be the data

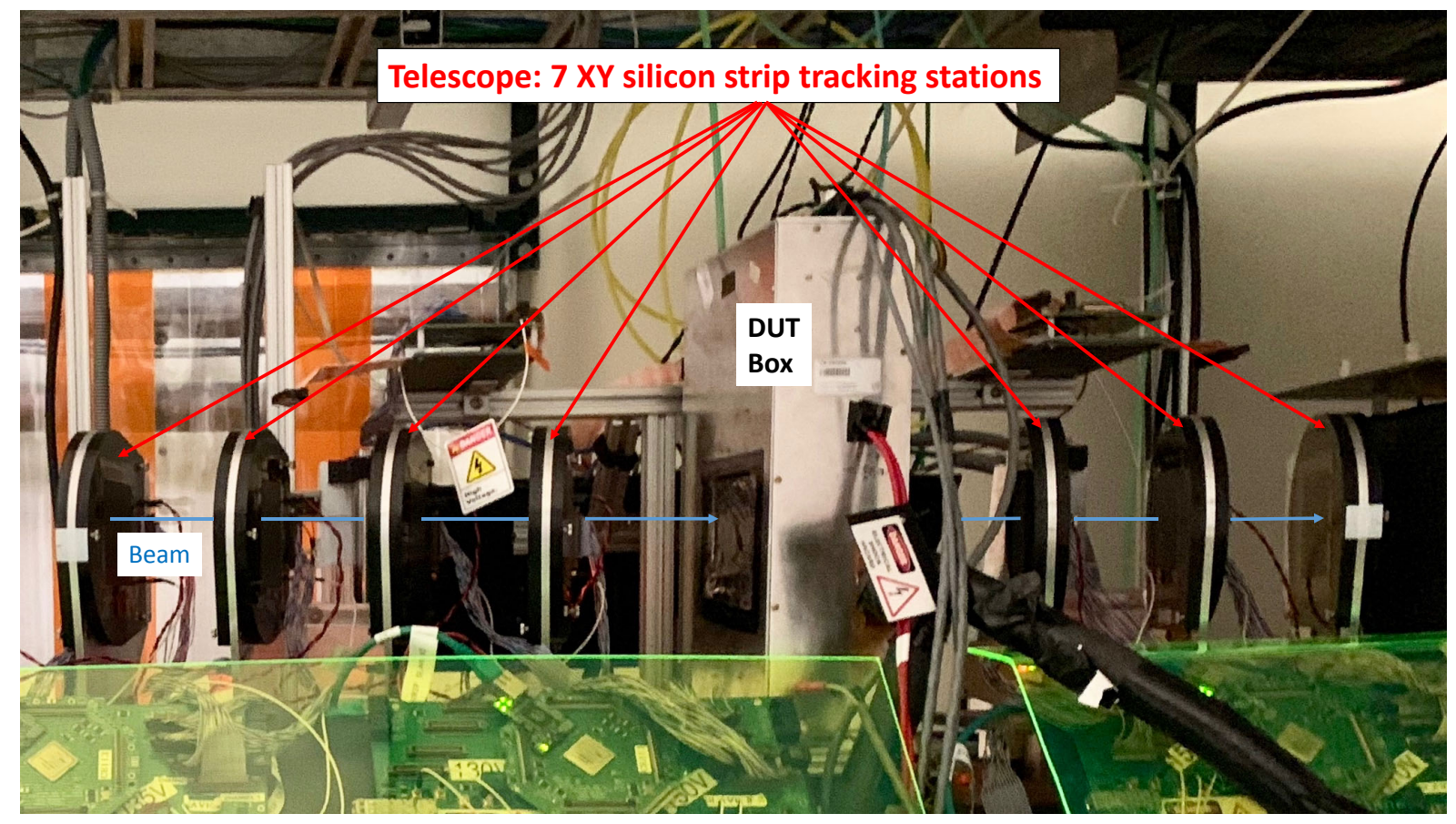

Figure 2: Photo showing the setup at the Fermilab Test Beam Facility (FTBF) for testing the UT sensors. The UT box containing the detector/sensor under test (DUT) is in the middle, and the 7 pairs of FTBF silicon-strip tracking stations are on either side. 
associated with a specific sampling of the SALT front end electronics, which operates at $40 \mathrm{MHz}$. Unfortunately, due to technical reasons that were never fully understood, MiniDAQ1 lost MEP data at a rate of about 0.5\%. (This problem does not affect the next generation DAQ board, MiniDAQ2.)

Techniques were developed within the offline software to recognize when MEP data was lost, and by using specific signatures in the data stream, resynchronization can generally be re-established. The software keeps track of the number of MEPs that are lost, and so, in principle, this can be accounted for in the offline analysis. This resynchronization is generally successful provided that the data rate is not too high. Once the data rate is too high, the signature used to recognize the loss of a MEP is no longer reliable, and the ability to re-synchronize the data is lost. This issue is rather technical in nature, but the important point is that this feature with MiniDAQ1 required that we set the threshold at about six times the CMS noise $\left(\sigma_{\text {noise }}\right)$, as opposed to a more reasonable value of about $3 \sigma_{\text {noise }}$ in order to maintain a relatively low hit rate. This is not an issue for the final system in LHCb, since the real DAQ system in LHCb will have a completely different DAQ, which will be interfaced directly with the host computer over PCIe, and will run firmware that will be able to handle the foreseen hit rate in LHCb. This higher-than-necessary threshold only affects this beam test.

Another complication with running at the FTBF is the different clocks that govern the two systems. The beam particles and the telescope readout are all synchronized to the Tevatron's $53 \mathrm{MHz}$ clock, whereas the SALT runs using a $40 \mathrm{MHz}$ internal clock onboard the MiniDAQ1. There are two issues here. First, there is no way to maintain time synchronization between the two systems running with independent clocks. Secondly, MiniDAQ1 samples the SALT front end every $25 \mathrm{~ns}(1 / 40 \mathrm{MHz})$, whereas the beam
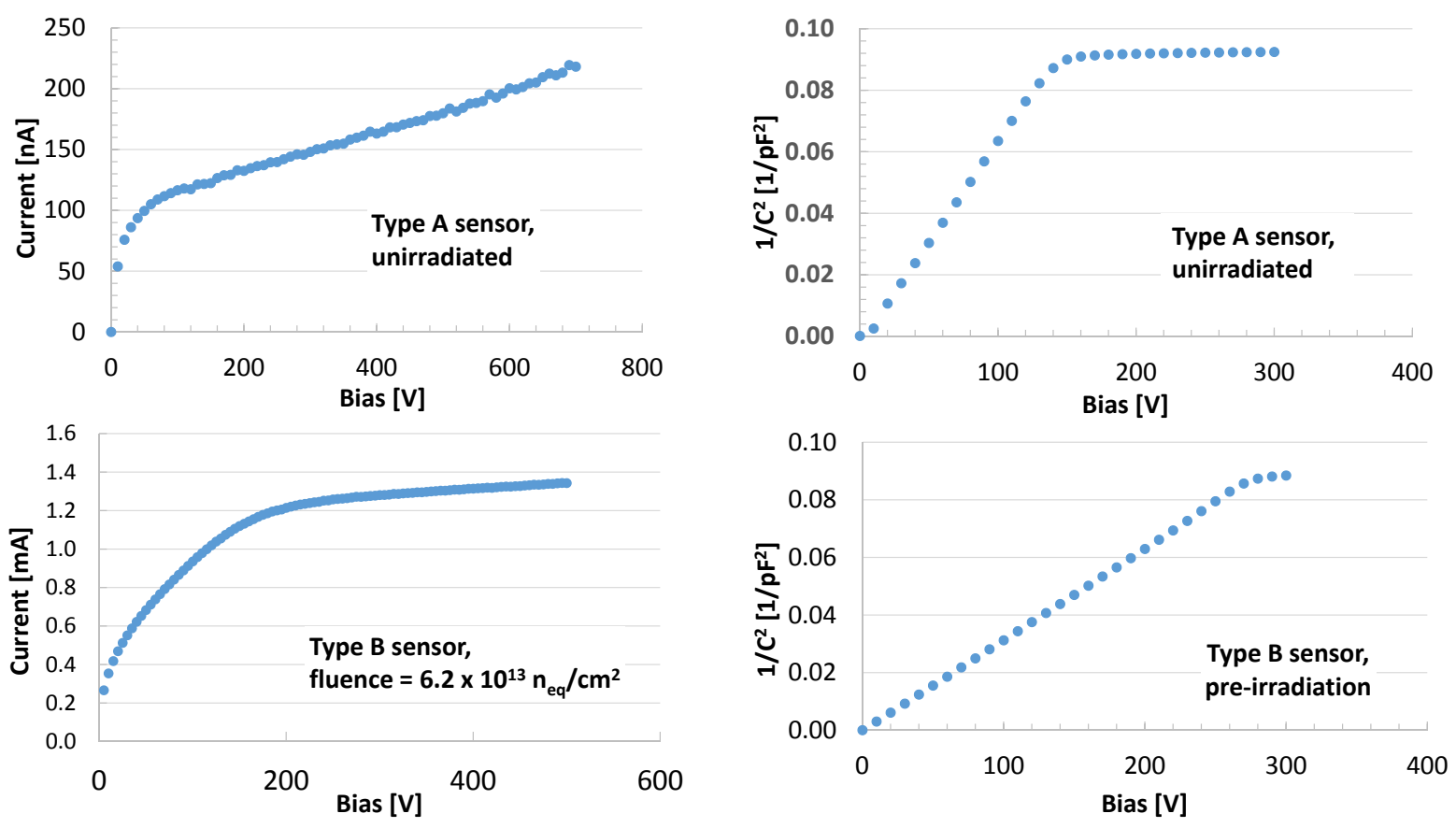

Figure 3: (Left) Current-voltage and (right) $1 / C^{2}$ versus the applied bias voltage for (top) the Type A sensor, and (bottom) the type B sensor. Heer, $C$ is the capacitance. 
particles are arriving at intervals of $19 \mathrm{~ns}(1 / 53 \mathrm{MHz})$, thus the beam particles will not, in general, arrive in time with the sampling of the SALT front end. To address these issues, the $53 \mathrm{MHz}$ clock from the Tevatron is put through a logic unit which produces a phase-locked clock at $3 / 4$ of $53 \mathrm{MHz}$, or $39.75 \mathrm{MHz}$. In advance of the testbeam, it was checked, and confirmed that the MiniDAQ1/SALT 3.0 could accept an external $39.75 \mathrm{MHz}$ clock, and run properly at the slightly lower frequency. From this point on, we refer to this clock as the $40 \mathrm{MHz}$ clock (although the actual value is about $39.75 \mathrm{MHz}$ ).

Even after this, there is still the issue that the beam particles arrive at intervals of 19 ns $(1 / 53 \mathrm{MHz})$, whereas the SALT front end is sampled with a period that is $4 / 3$ larger, or about every $25 \mathrm{~ns}$. It is not possible to have have every beam particle arrive in phase with the $40 \mathrm{MHz}$ sampling clock. In fact, because of the factor of $3 / 4$, we expect only one out of every four beam particles to be in-phase, or almost in-phase, with the $40 \mathrm{MHz}$ clock. The situation is depicted in Fig. 6. The top line depicts a $40 \mathrm{MHz}$ clock, and the second line shows a $53 \mathrm{MHz}$ clock, where the first falling edges are aligned. One sees that the second, third and fourth falling edges of the $53 \mathrm{MHz}$ clock are not in phase with the 40 $\mathrm{MHz}$ clock, but the fifth one is back in phase. The green circles represent beam particles that would be in phase with the sampling of the SALT front end. The red circles indicate beam particles that are far out of time, and the blue ones represent somewhere in between. Only beam particles represented by the green circles would have the maximum charge collected. The red would have the least, and the blue somewhere in between. For any pair of UT and telescope data runs, one cannot control the relative phase between the $40 \mathrm{MHz}$ and $53 \mathrm{MHz}$ clocks. The third through sixth lines show a similar situation, except the phase of the $53 \mathrm{MHz}$ clock is shifted incrementally to represent different possible offsets. In these cases, none of the beam particles are perfectly in phase with the $40 \mathrm{MHz}$ sampling

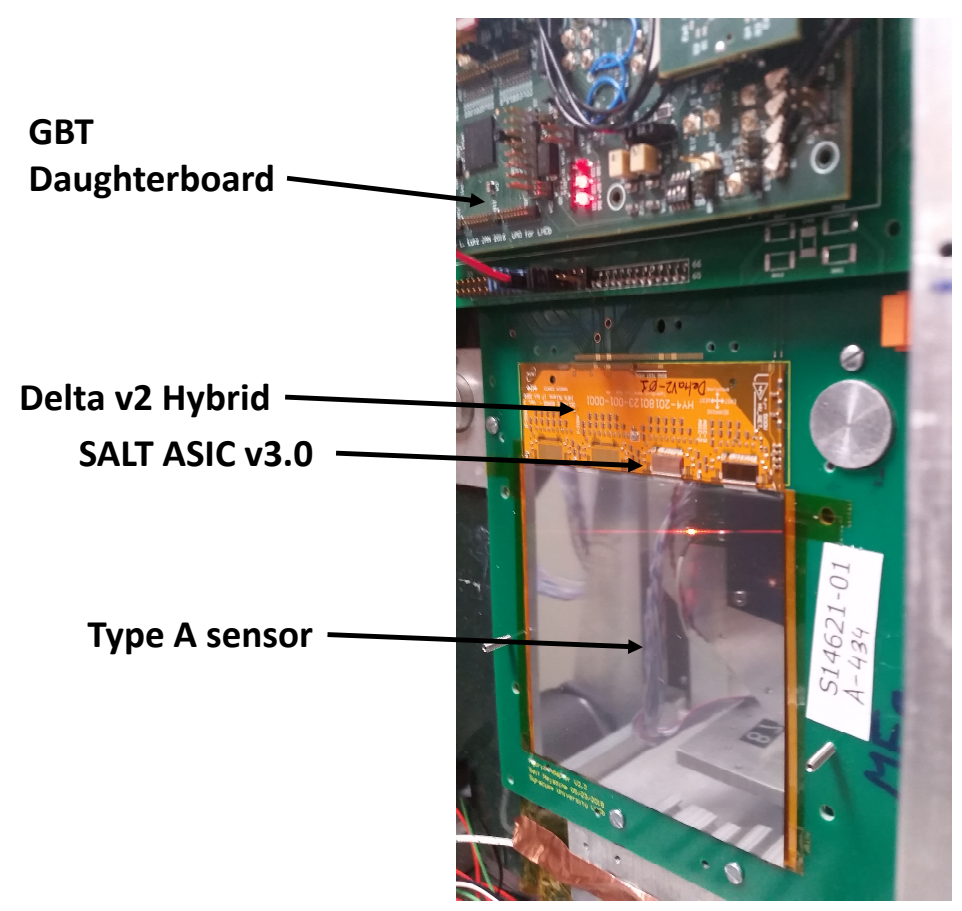

Figure 4: Photo showing the Type A module that was tested in the Fermilab test beam. 
clock. However, it can be shown that in the worst case scenario, there is a beam particle no further than 3 ns away from the edges labeled FE sampled. Given the pulse shape after the shaper in SALT 3.0, a 3 ns offset should not reduce the detected signal more than a few percent from the optimal value. Because of the repetitive pattern, we can categorize the beam particles into four unique time bins $(\mathrm{TB}) \mathrm{TB}_{i}=\left(n_{\text {clock }}^{53 \mathrm{MHz}}\right) \bmod (4)$, where $n_{\text {clock }}^{53 \mathrm{MHz}}$ is the number of cycles of the $53 \mathrm{MHz}$ clock, and the $\bmod (4)$ represents modulo 4. In this way, $\mathrm{TB}_{0}$ picks out clocks $0,4,8, . ., \mathrm{TB}_{1}$ selects clocks $1,5,9, \ldots$ and so on. We then choose the best time bin as the one which has the largest charge collected.

All data were taken with the UT sensor plane perpendicular to the direction of the beam particles, apart for possible a very small rotation of the UT test box relative to the beam. The angular spread of the beam is small, well below $1 \mathrm{mrad}$.

\subsection{Threshold settings for UT}

The initial setup of the UT system showed that there was a very high level of noise in the testbeam environment. Total noise in the system was approximately 7 ADC counts. A large number of grounding configurations were tried, and the best performance achievable in the limited time available yielded a total noise of about 3.5 ADC counts, and a common-mode subtracted noise level of about 1 ADC count. It was also observed that the total noise decreases substantially when the UT box was moved away from the telescope, leading to the conclusion that the UT was picking up coherent noise from the Fermilab telescope hardware. Due to the issues with the MiniDAQ1 discussed above, most of the data were taken at a threshold of 6 , which corresponds to $A D C \geq 7$. Some data was taken at a threshold of 5 , corresponding to $\mathrm{ADC} \geq 6$. We emphasize that this threshold
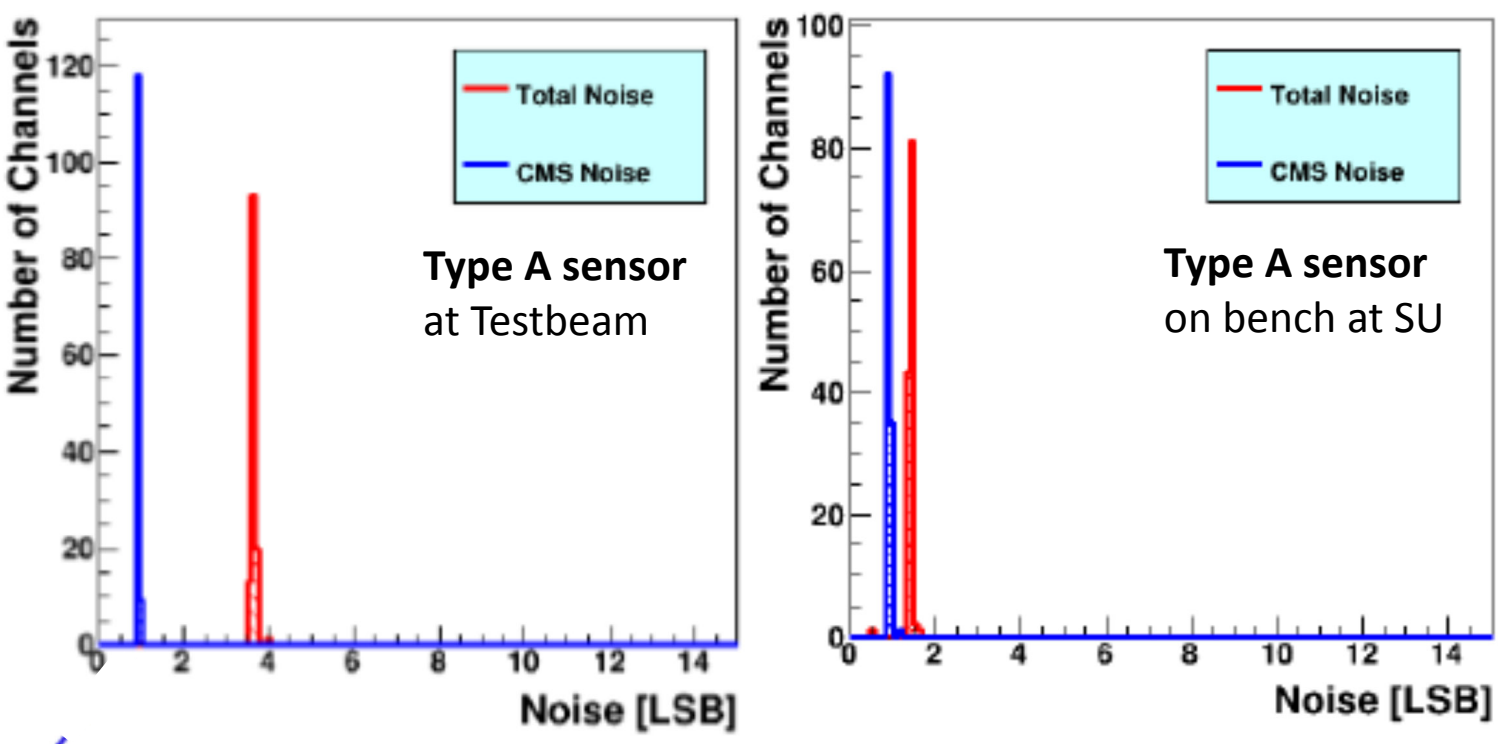

Figure 5: Total noise and common-mode subtracted noise for the Type A sensor (left) while in the FTBF testbeam and (right) after the testbeam on the bench at Syracuse. 


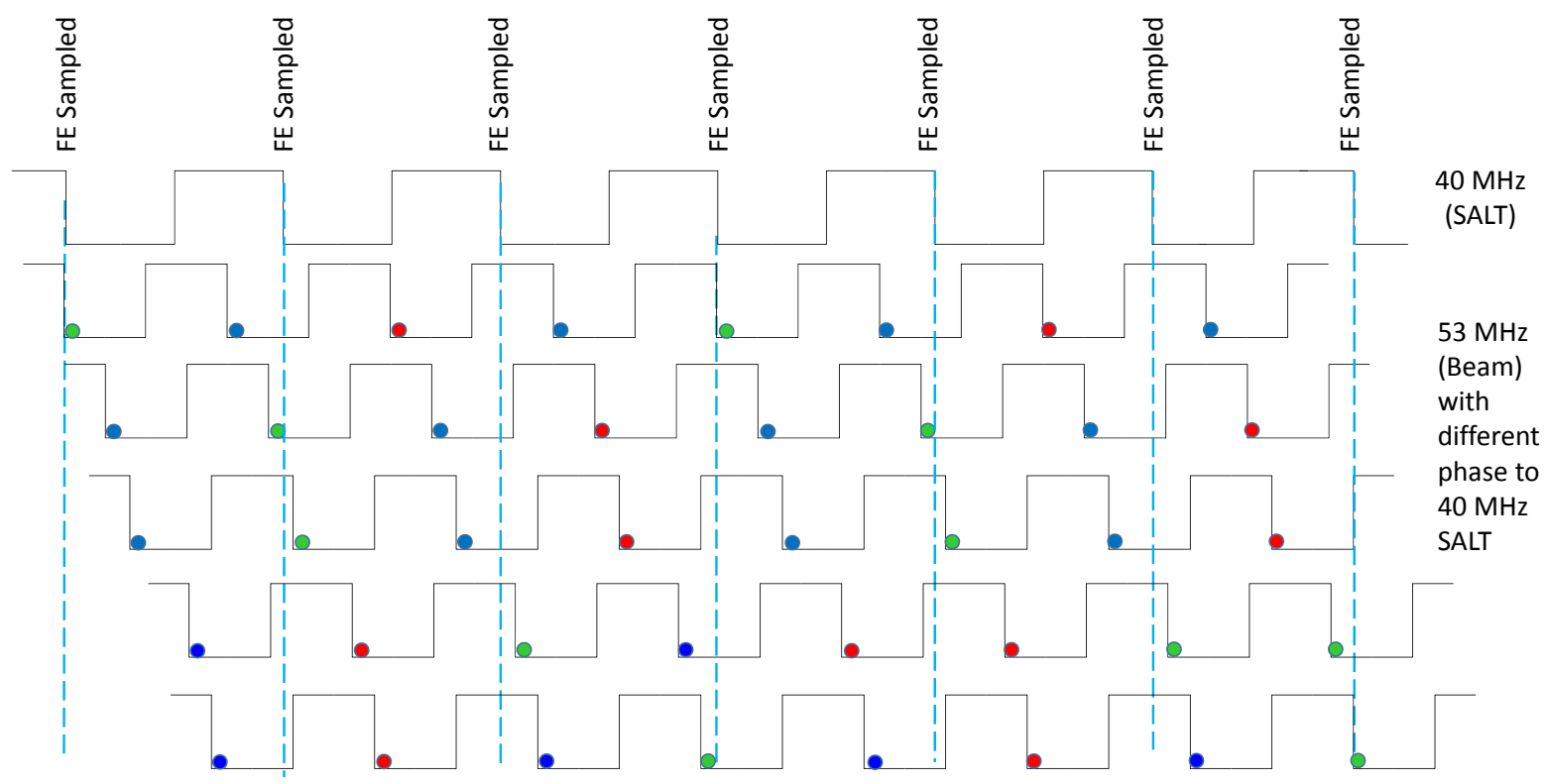

Figure 6: Cartoon showing the $40 \mathrm{MHz}$ clock of the SALT (top), and a $53 \mathrm{MHz}$ clock with several different phases relative to the $40 \mathrm{MHz}$ clock. In the second line, the first falling edges of the two clocks are aligned, and thereafter the $53 \mathrm{MHz}$ clock is incrementally shifted to the right.

is much higher than what we expect to run at in the real system. In the real system in LHCb, we expect to run with the threshold at 3 or 4 , corresponding to about $3-4 \sigma_{\text {noise }}$.

The results presented in this note are limited to the runs with the lower threshold of $\mathrm{ADC} \geq 6$. With these thresholds, we find that some channels have extremely high noise rates. For those channels, the ADC threshold is increased one unit at a time until the channel's hit rate is no more than four times the average hit rate. Up to 5 passes are performed, so thresholds can go up to 11, but no more. About 10-20\% of channels had these higher levels of noise, and typically they have thresholds of $\mathrm{ADC} \geq 7$ or 8 , although a few may require $\mathrm{ADC} \geq 9$ or 10 . In bench tests of the beam test modules after the test beam, we could not reproduce the higher levels of noise on these channels.

\section{$2.2 \quad$ Alignment}

In our setup, we define the coordinate system to be such that the beam axis defines the $z$ direction, the horizontal direction provides the $x$ coordinate and the vertical direction is assigned to be the $y$ direction. The UT sensors are strip sensors, where the strips run parallel to $y$, and therefore provide precision measurements in the $x$ direction. For the studies presented here, we do not need precision alignment. The UT sensors are aligned to account for translations along $x$ and rotations around the $z$ axis. 


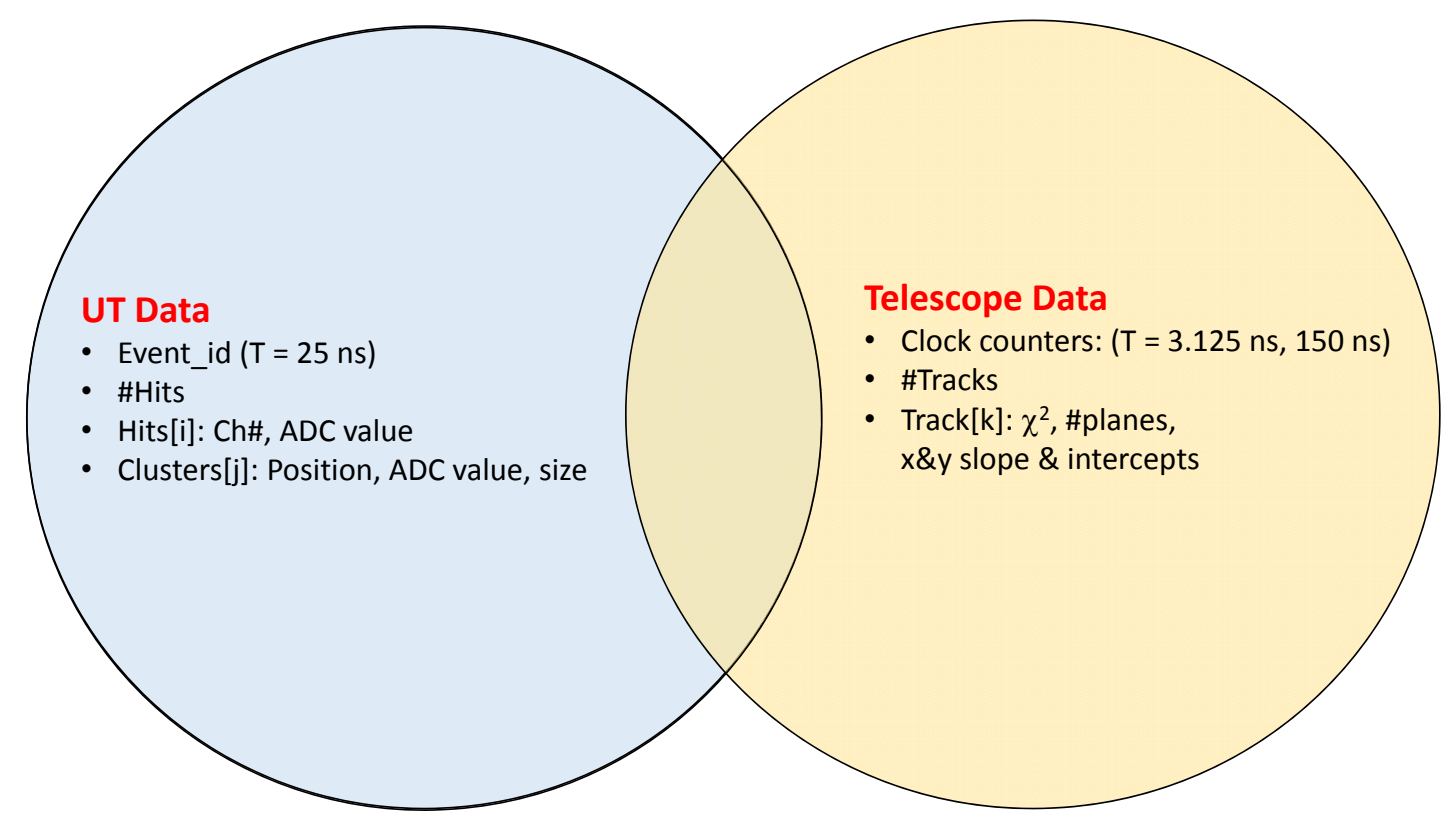

Figure 7: Venn diagram showing the relationship between the UT and tracking data sets. along with the key quantities that are provided for offline analysis.

\section{Analysis of the data}

The data from the UT and the telescope are each output in the form of independent ROOT files. For the analysis to proceed, we must match the events from the two systems in both time and space. A Venn diagram showing the relationship of the two data sets is shown in Fig. 7. Also shown are the key quantities that are stored for offline use. The overlap region shows the common set of events where a particle passed through both the telescope and the UT DUT, and produced a track in the former and at least one hit in the latter. These form a subset of the total tracks or hits recorded by each system. Before analysis can be done, the telescope track from event $n$ has to be matched to the correct hit in event $m$ from the UT system. Once that is done, the analysis can proceed.

\subsection{Matching of the UT and telescope data}

The matching of events of the two systems relies on the event_id in the UT data and the $150 \mathrm{~ns}$ clock counter from the telescope. The event_id in the UT increments at 40 $\mathrm{MHz}$, while the telecope has counters at both $6.667 \mathrm{MHz}$, and $320 \mathrm{MHz}$. The $6.667 \mathrm{MHz}$ telescope counter is used to perform the time matching to the UT system, since tracks are well separated in time. To match the frequency of the $6.667 \mathrm{MHz}$ counter to that of the UT system the telescope counter is multiplied by 6 , effectively making it a $40 \mathrm{MHz}$ counter (same as UT system). We refer to the $40 \mathrm{MHz}$ counter from the UT system as 
$C_{\mathrm{UT}}$ and that from the telescope as $C_{\text {tel }}$. Between the two systems, there is an arbitrary, run-dependent time offset, or equivalently, an offset between their counters. This offset needs to be determined, and once it is determined and applied, events with tracks in both systems should be correlated in time. Namely, after the offset is determined, there should be a definite excess of events near zero in the difference $C_{\mathrm{UT}}-C_{\text {tel }}$.

The correlation in time is done by first locating the beginning and end of the first spill seen by each system. Care was taken to ensure that both systems started their DAQs together, so that the first spill of particles seen by the UT was also the first spill seen by the tracking system. For the UT system, there is a steady stream of noise hits even when there are no real tracks. The signature of the beginning of the spill is a step function increase in the number of hits per unit time. An algorithm is used to find that leading edge. In the telescope system, it is simpler, because the arrival of the spill is characterized by going from zero tracks per second, to some finite number. Once the first spill has been found in each system, the end of the spill is then known to be $4.2 \mathrm{~s}$, or $(4.2 \mathrm{~s})(40 \mathrm{MHz})=168 \times 10^{6}$ counts later. Once this has been done, we have a pair of counter intervals for the two systems: $\left[C_{\mathrm{UT}}^{\mathrm{start}}, C_{\mathrm{UT}}^{\mathrm{end}}\right]$ and $\left[C_{\mathrm{tel}}^{\mathrm{start}}, C_{\mathrm{tel}}^{\mathrm{end}}\right]$. The difference in $C^{\text {start }}$ between the spill as seen by the two systems' counters is then applied as an offset, which brings the two system's counters close to being in phase. However, this first estimation of $C^{\text {start }}$ is a rough estimate, and has a precision of about $10 \%$ of the time interval itself, or $\sim 10^{7}$ counts. This is mainly due to the fact that the recognition of the step increase in the UT hit rate requires us to integrate over about $10 \%$ of the spill, since the track intensity is fairly low $(\sim 5 \mathrm{kHz})$ compared to the noise rate.

To determine the precise time offset, an iterative procedure is performed. The method relies on the fact that if we loop over all $\left(C_{\mathrm{UT}}, C_{\mathrm{tel}}\right)$ pairs from the respective systems within the first spill, and compute $\delta C \equiv C_{\mathrm{UT}}-C_{\text {tel }}$ (after the rough offset discussed above is applied), a sharp spike must appear somewhere in the $\delta C$ spectrum, although not necessarily at zero due to the rough estimate of $C^{\text {start }}$. The spike corresponds to both systems responding to the same beam particle. To determine a more precise value of the offset between the two systems, the difference $\delta C$ is put into a histogram with a very wide range to cover all reasonable values of the residual offset. The position of this spike is found, the corresponding counter offset is determined, and then the counter offset is updated with a new offset. This procedure is then repeated with a narrower time window, until we have determined the mean time offset to within about 1 count.

Figure 8 shows the results of this iterative procedure for a single spill of the unirradiated Type A sensor, taken with a bias voltage of $250 \mathrm{~V}$. The top row shows the difference $C_{\mathrm{UT}}-C_{\text {tel }}$ between the two systems in each of the five iterations. The bottom row shows the spacial difference $\Delta x=x_{\mathrm{UT}}-x_{\text {track }}$, where $x_{\text {track }}$ is the projected track position at the location of the DUT and $x_{\mathrm{UT}}$ represents the position of a cluster. A cluster is a contiguous groups of strips, each above the strip threshold of 5 ADC counts. The position of the cluster is obtained from the charge-weighted average of the contributing strips. One can see in the top left plot the spike at about $20 \times 10^{6}$. Because the two systems are not (necessarily) aligned in space, an arbitrary offset in $\Delta x$ is also expected. In the second iteration (second column), a correction to $C^{\text {start }}$ is applied, and the considered time window for matching two systems is narrowed. As we effectively zoom in, we see the correlation in time is more pronounced and closer to zero. Again, a time offset is obtained from the second iteration, and a narrower time-matching window is applied. The spacial correlation is typically not seen in the first and second iterations, since the combinatorics 

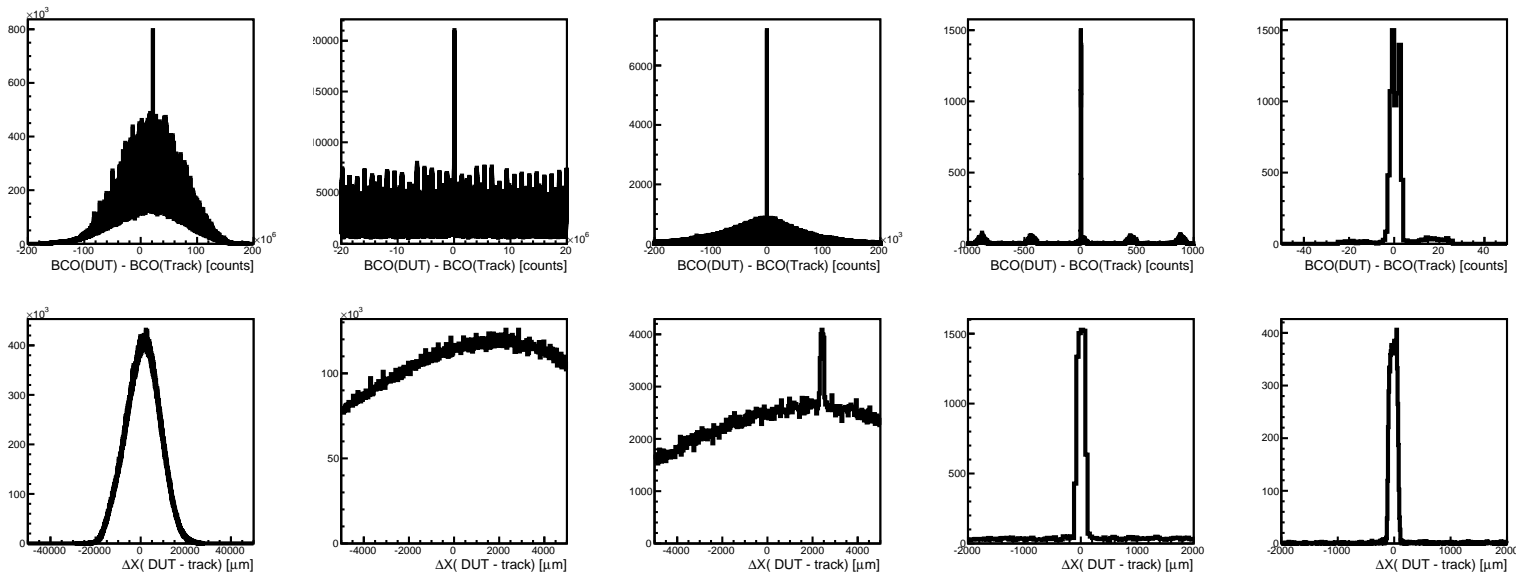

Figure 8: Results of the temporal and spacial alignment procedure, as described in the text, for the Type A sensor at a bias voltage of $250 \mathrm{~V}$. In the time plots, the units are $25 \mu \mathrm{s}$. The peaks seen every $11.2 \mu \mathrm{s}$ in the fourth plot in top row is due to the way the beam is created and extracted from the main injector at the Tevatron [7].

are too large to see the excess. In the third iteration, we begin to see not only the time matching, but also the spacial matching. We can see that the two systems are offset by about $2.4 \mathrm{~mm}$. After the third iteration, we apply both a time offset and a spacial offset, to bring the two systems into temporal and spacial alignment. The final alignment in time and space is shown in the fifth column. Once the two systems are aligned in time and space, a combined ROOT file with all tracks, and any matching hits are written out for further analysis for all spills.

\subsection{Results on the Type A sensor}

With the systems aligned in time and space, and the thresholds in the DUT set, performance of the UT sensors and SALT 3.0 ASIC can be studied. We first present results on the unirradiated Type A sensor.

As mentioned previously, events are grouped into one of four time bins in order to deal with the different frequencies of beam particles $(53 \mathrm{MHz})$ versus the SALT ADC sampling (40 MHz). Figure 9 shows the ADC distributions for each of the four time bins for the Type A sensor at $300 \mathrm{~V}$ bias. Comparing the four distributions, it is clear that time bin $\mathrm{TB}=3$ provides the largest signal as well as the highest efficiency. We see that $\mathrm{TB}=0$ and 2 have somewhat lower mean values and efficiencies, and $\mathrm{TB}=1$ has the lowest charge collected and efficiency. This pattern is consistent with expectations due to the different frequencies between the beam particles and the SALT3 sampling and an arbitrary relative phase between the two, as shown in Fig. 6 .

All runs investigated show a similar behavior, namely there is a best time bin, a very bad time bin, and two that are somewhere in between. Each run, in general, has its own best time bin, and unless otherwise noted, results are quoted from the best time bin. In plots where all four time bins are shown, it should be kept in mind that the most relevant one for this run is time bin $\mathrm{TB}=3$.

Figure 10 shows the (left) $\Delta x$ distribution and (right) number of strips in the cluster, 

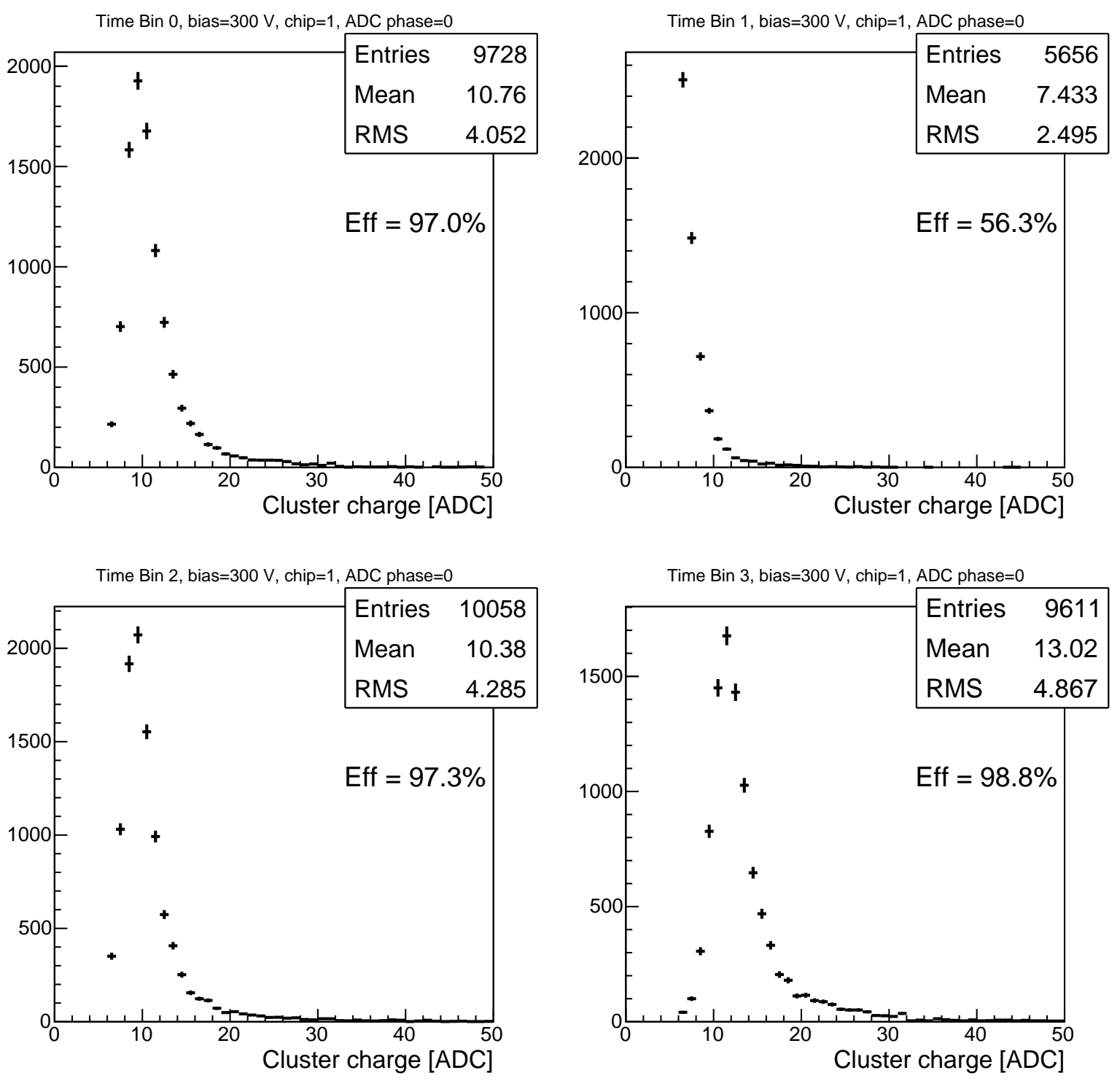

Figure 9: Distributions of collected charge (in ADC counts) for a single run of the Type A sensor at $300 \mathrm{~V}$ bias, for the four time bins. See text for details.

for clusters matched within $1 \mathrm{~mm}$ of a track (approximately 5 times the strip pitch). The UT sensor bias voltage is $300 \mathrm{~V}$. The vertical red lines show the width of a strip. Almost all hits are within \pm 0.5 of the strip pitch, and most hits are single-strip hits.

Figure 11 shows the cluster charge for clusters matched to tracks at $300 \mathrm{~V}$ bias voltage for the best time bin $(\mathrm{TB}=3)$. Overlaid is a fit to a Landau function convolved with a Gaussian resolution function and the fit parameters are shown. The peak of the Landau is at 11.1 ADC counts. Due to a design issue with SALT 3.0, strips with ADC value larger than 31 are lost in sensors that have electron charge carriers (p-in-n), which introduces a small inefficiency. The issue does not affect sensors where the majority carriers are holes (n-in-p). By comparing the fraction of clusters with ADC $>31$ between the p-in-n sensor, which is affected by the design issue, and the n-in-p, which is not affected, we estimate the inefficiency in the p-in-n sensors is at the level of $(0.1-0.2) \%$. It is expected 
Bias $=300 \mathrm{~V}$, chip $=1$, ADC phase $=0$

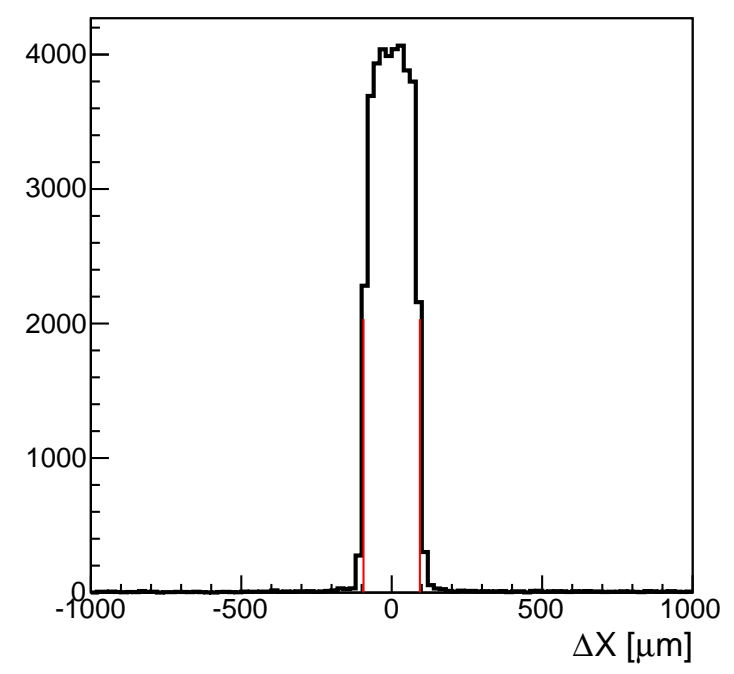

Bias $=300 \mathrm{~V}$, chip $=1$, ADC phase $=0$

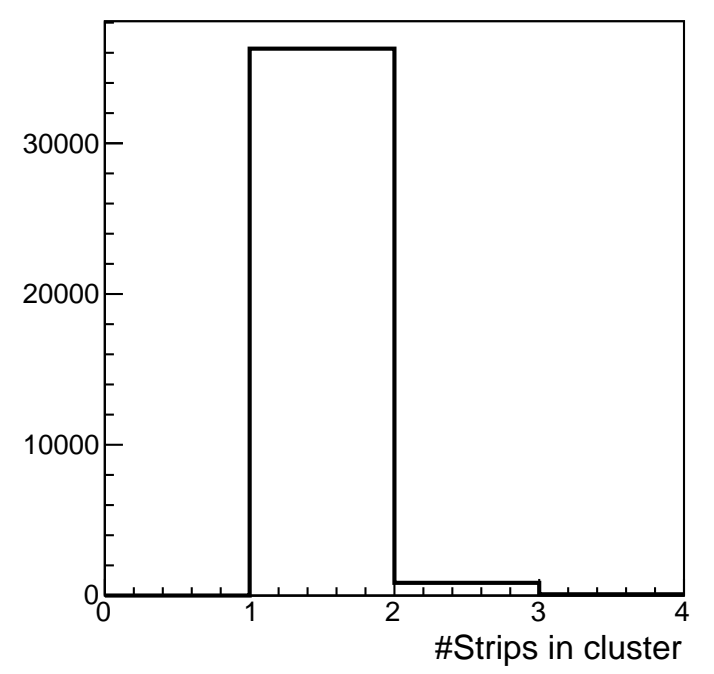

Figure 10: Distribution of (left) $\Delta x=x_{\mathrm{UT}}-x_{\text {track }}$ and (right) number of strips in a cluster, for clusters within $1 \mathrm{~mm}$ of a telescope track for the type A unirradiated sensor at $300 \mathrm{~V}$ bias.

that in $320 \mu \mathrm{m}$ of silicon, the most probable number of electron-hole pairs generated is about $25000^{3}$. Taking the most-probable-vale of 11.1 ADC to correspond to this charge, we estimate that $1 \mathrm{ADC}$ count is equivalent to approximately 2250 electrons.

We have also investigated the charge collected and efficiency as a function of the bias voltage. For the efficiency evaluation, only events with a single reconstructed track with at least 10 out of a maximum 14 possible hits are selected. The efficiency is defined as the fraction of tracks that have a cluster within $\pm 1 \mathrm{~mm}$ of the projected track position at the DUT, although almost all clusters are within $\pm 150 \mu \mathrm{m}$, as seen in Fig. 10. Figure 12 shows the results of the efficiency and charge collection as a function of the bias voltage applied. The charge collection is quantified in terms of the peak position of the fit to the charge collected using a Landau convolved with a Gaussian function. The efficiency is evaluated as described above. The efficiency is seen to reach its maximum value, about $99 \%$, for bias voltages above $150 \mathrm{~V}$. The peak of the Landau fit increases and plateaus at about 11.4 at a voltage of about $250 \mathrm{~V}$. Given the common-mode subtracted noise is about 0.95 ADC counts, the signal-to-noise ratio $(\mathrm{S} / \mathrm{N})$ is about 12 . This is similar to that found in earlier beam tests of Type A sensors using Beetle chips [6].

As the type A sensor is fully efficient by $300 \mathrm{~V}$, we investigate some additional properties at this voltage. Figure 13 shows the average cluster size as a function of the interstrip position. For these plots, zero in the relative strip (or interstrip) position corresponds to a track intercepting the UT sensor at the midpoint between any two adjacent strips, whereas $-0.5(0.5)$ corresponds to the track pointing directly at the strip to the left (right) of the middle. Every track/cluster entry is projected into this range, integrated over all track/cluster matches. Although all four time bins are shown, the focus should be on time bin 3, as the others are not optimally aligned with the SALT $40 \mathrm{MHz}$ ADC sampling

\footnotetext{
${ }^{3}$ This value is obtained using \# $\mathrm{e}$-hole pairs $=(388 \mathrm{eV} / \mu \mathrm{m})(0.73)(320 \mu \mathrm{m}) /(3.62 \mathrm{eV} / \mu \mathrm{m})$, where the values come from Fig. 33.9 in Ref. [10], and we assumed $\beta \gamma=100$ and have used $3.62 \mathrm{eV} / \mu \mathrm{m}$ as the average energy deposition of a minimum ionizing particle in silicon.
} 


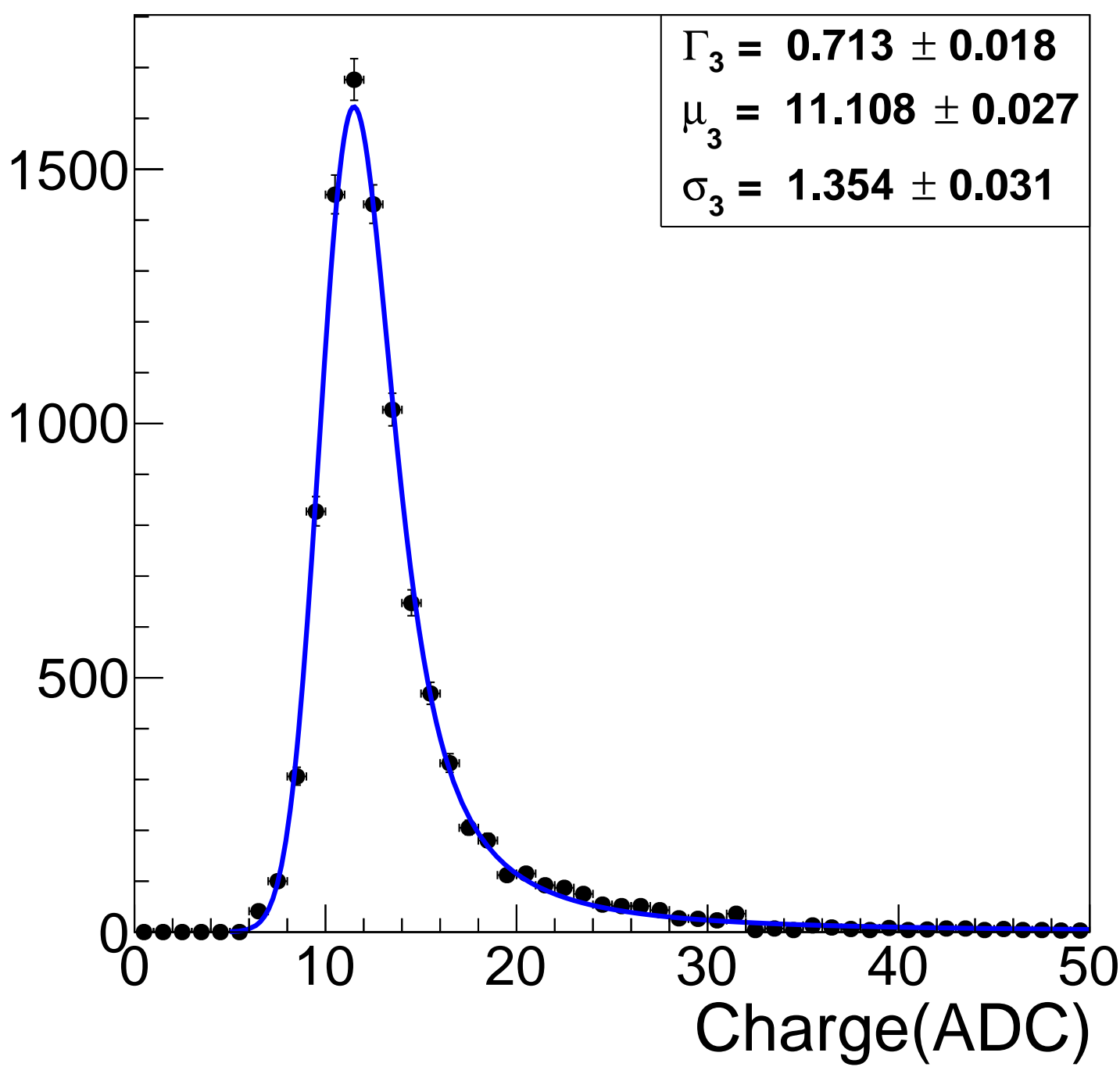

Figure 11: Distributions of collected charge (in ADC counts) for the Type A sensor at $300 \mathrm{~V}$ bias, for the best time bin. See text for details.

clock. It is seen that the average cluster size increases and reaches about 1.1 when the track passes through the middle between two strips. This increase is expected, since at the middle there is a greater chance for splitting the charge and forming a two-strip cluster. When the track hits directly on top of a strip, the average cluster size is very close to 1 . This narrow region of charge sharing occurs due to the electric field profile and diffusion within the bulk of the silicon 4 .

The average charg $\biguplus^{5}$ collected versus the interstrip position is shown in Fig. 14. The charge collected is seen to be uniform across the strip, even when the track is near the

\footnotetext{
${ }^{4}$ See Ref. [1], and in particular Figure 15(a), which shows the simulated drift paths of electrons and holes.

${ }^{5}$ Here, for convenience the average charge is shown, and not the peak of the Landau. Since they are correlated and we are mainly interested in trends, this is not an issue.
} 


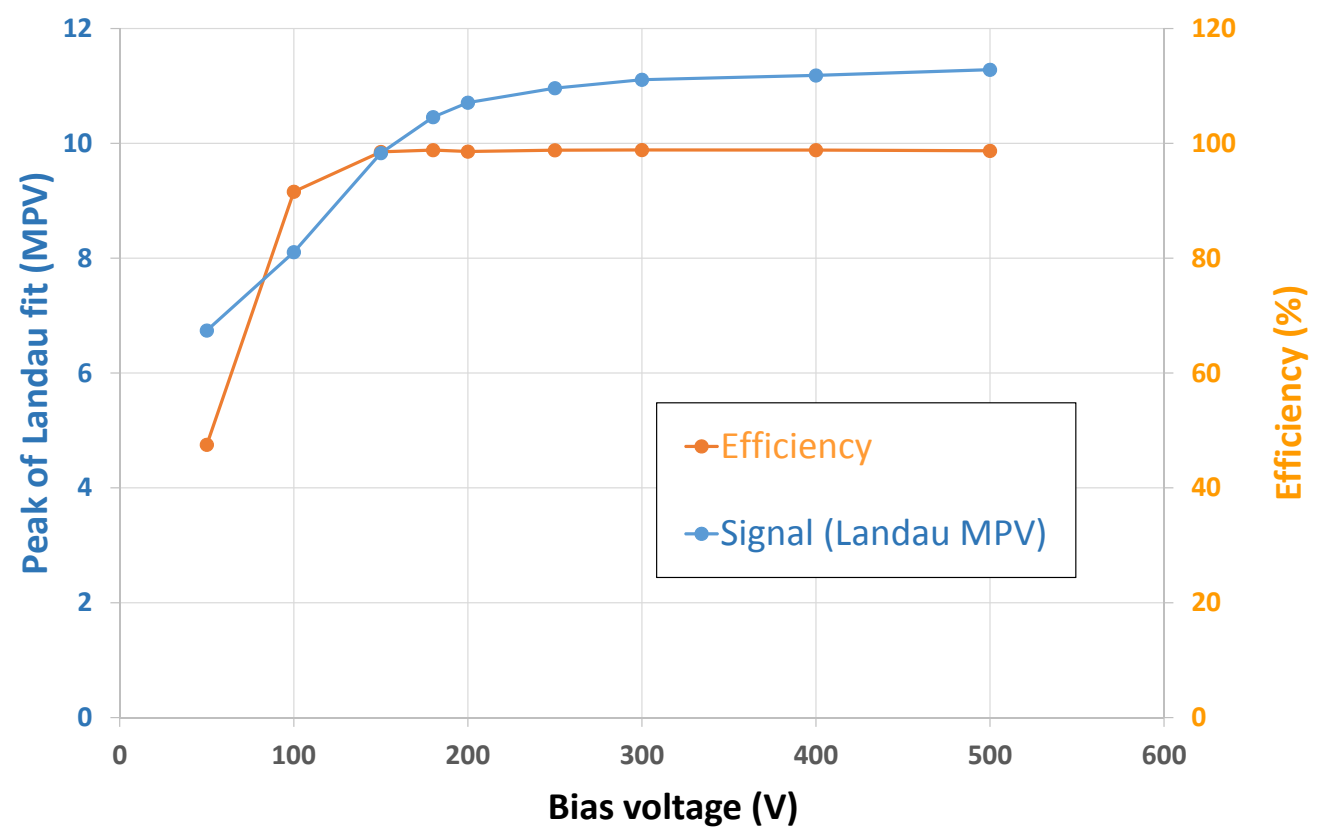

Figure 12: Peak of the Landau fit and efficiency of the Type A unirradiated UT sensor versus the applied bias voltage.

middle and charge is shared between the strips.

Figure 15 shows the efficiency as a function of the interstrip position. As with the collected charge, the efficiency is seen to be flat versus the interstrip position, indicating even when the track points in the middle between the two strips, and charge is shared between the two strips, there is little or no loss of efficiency.

The studies performed on the type A sensor indicate that it meets the needs of the LHCb upgrade. Most of the type A sensors receive very low irradiation, and therefore we do not expect a significant degradation in the signal-to-noise performance over the life of the sensor. An efficiency of about $99 \%$ is achieved in this beam test. Due to the issue of packet loss in MiniDAQ1, we know about $0.5 \%$ comes from this source. We therefore find that the efficiency of the Type A sensor in the testbeam is at least $99.5 \%$. 

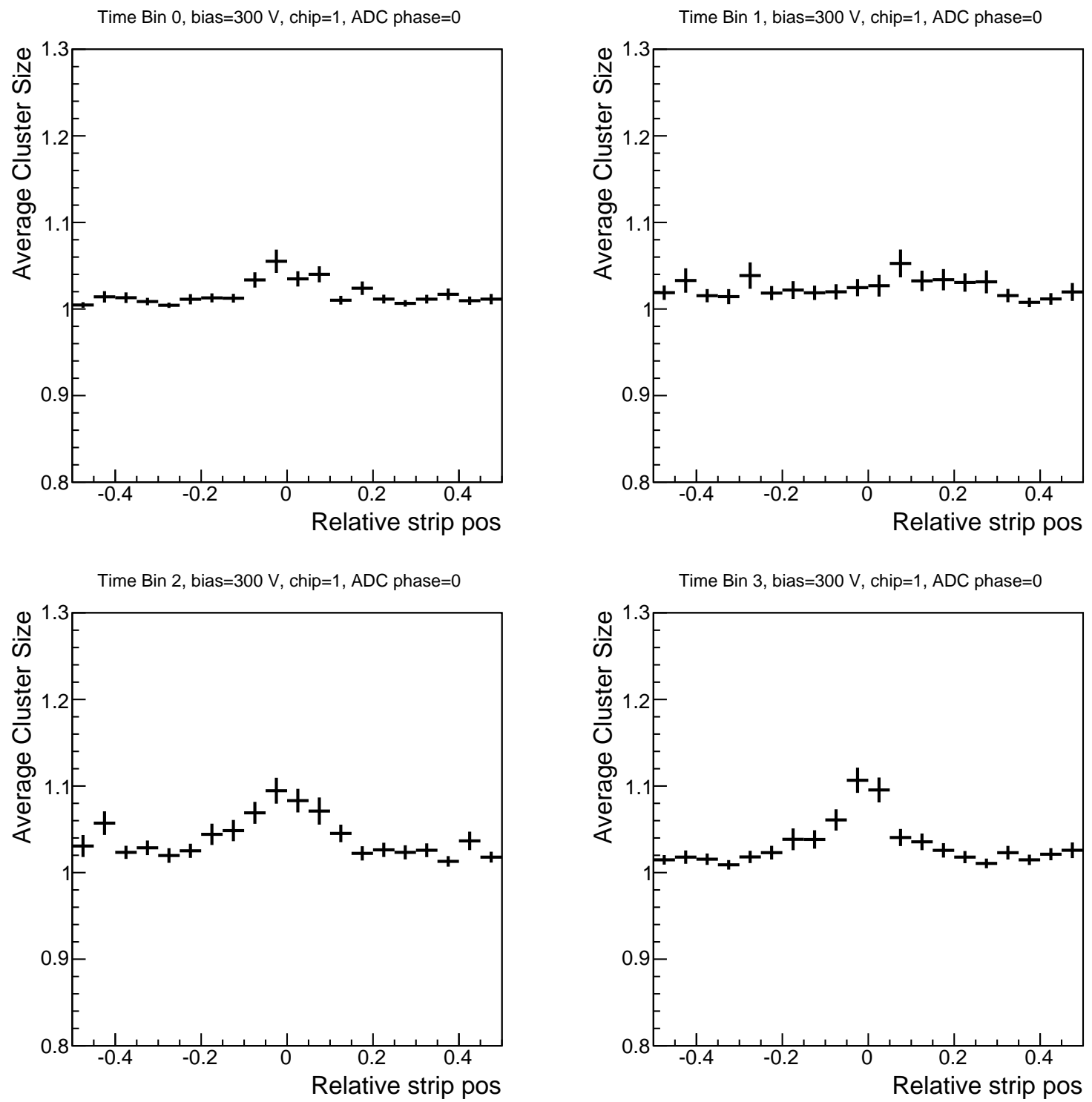

Figure 13: Average cluster size versus the interstrip position for each of the four time bins. The most relevant one is time bin $=3$ (bottom right). Zero indicates the track intercepts the DUT midway between two adjacent strips. 

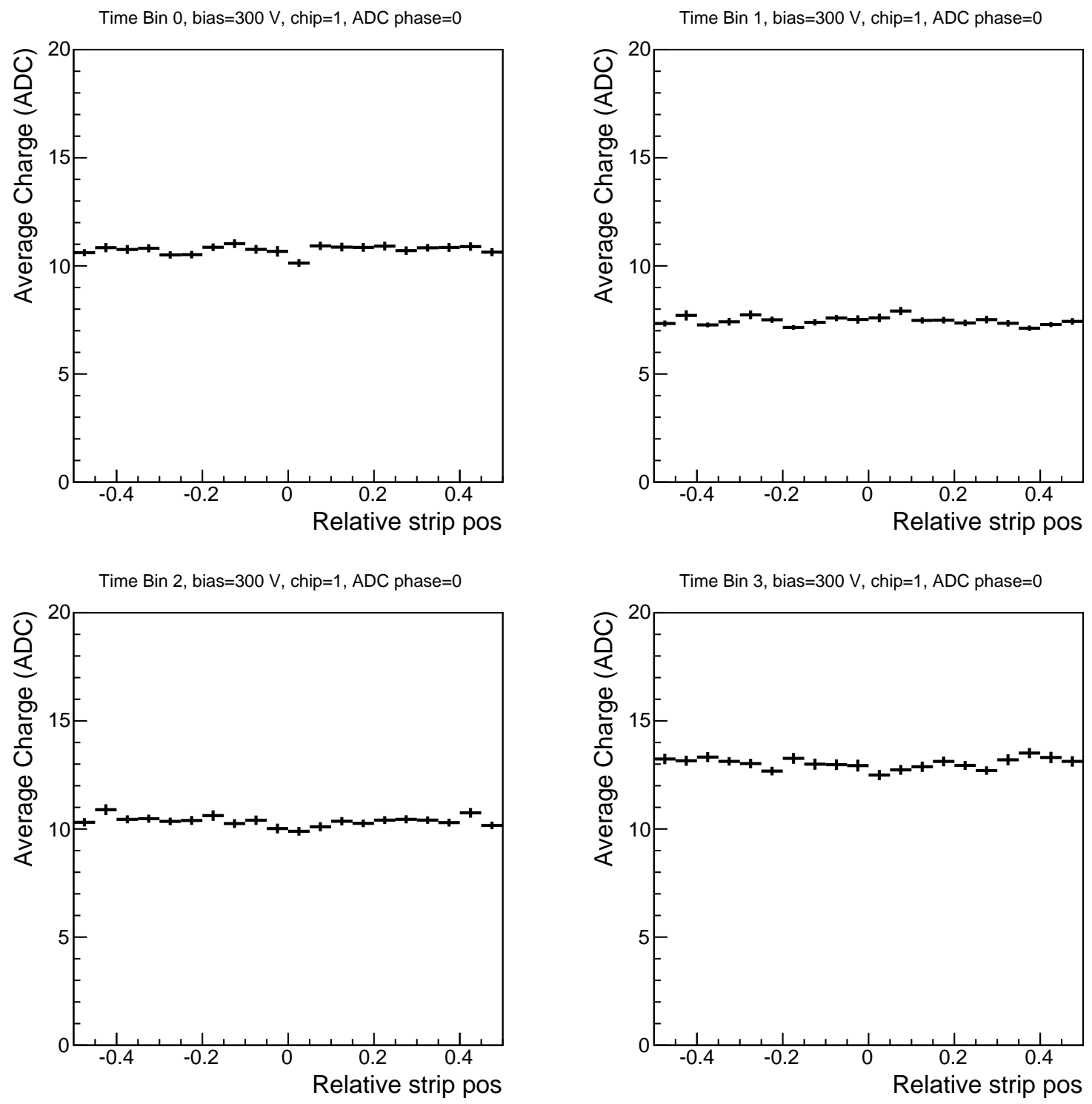

Figure 14: Average cluster charge (in ADC) versus the interstrip position for each of the four time bins for the Type A sensor. The most relevant one is time bin $=3$ (bottom right). 

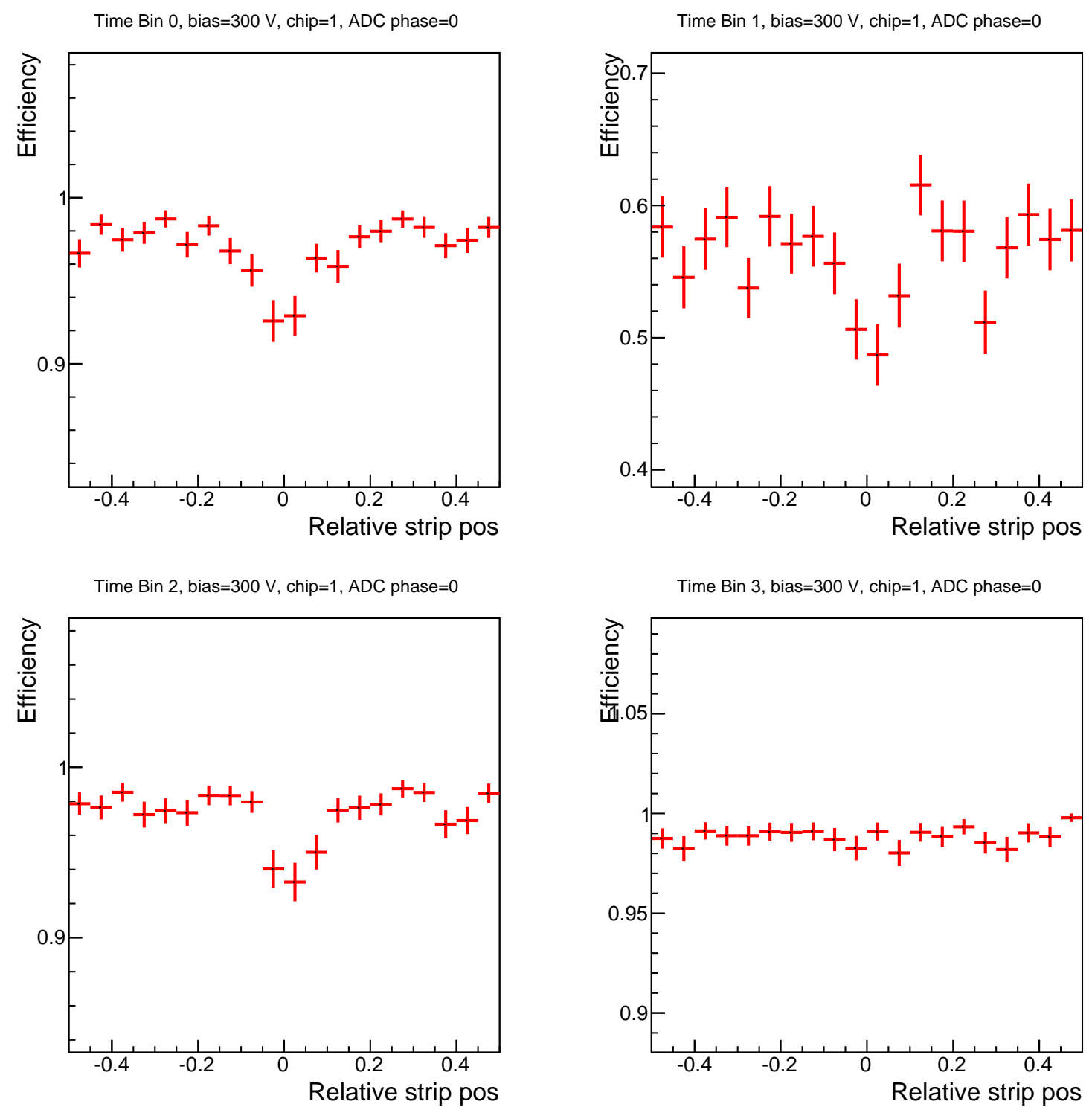

Figure 15: Efficiency versus the interstrip position for each of the four time bins for the Type A sensor. The most relevant one is time bin $=3$ (bottom right). 


\subsection{Results for the the Type B sensor}

Similar studies were performed with the Type B sensor that was irradiated to $6.2 \times 10^{13}$ $n_{e q} / \mathrm{cm}^{2}$, which is about twice the maximum expected fluence anticipated for Type B sensors in the UT. The threshold for the results presented here is also ADC $\geq 6$, which is the same as for the type A sensor results. The results of the bias scan are shown in Fig. 16. It is seen that the efficiency reaches a plateau of about $94 \%$, while the charge collected has a most probable value of about 10.3 ADC.

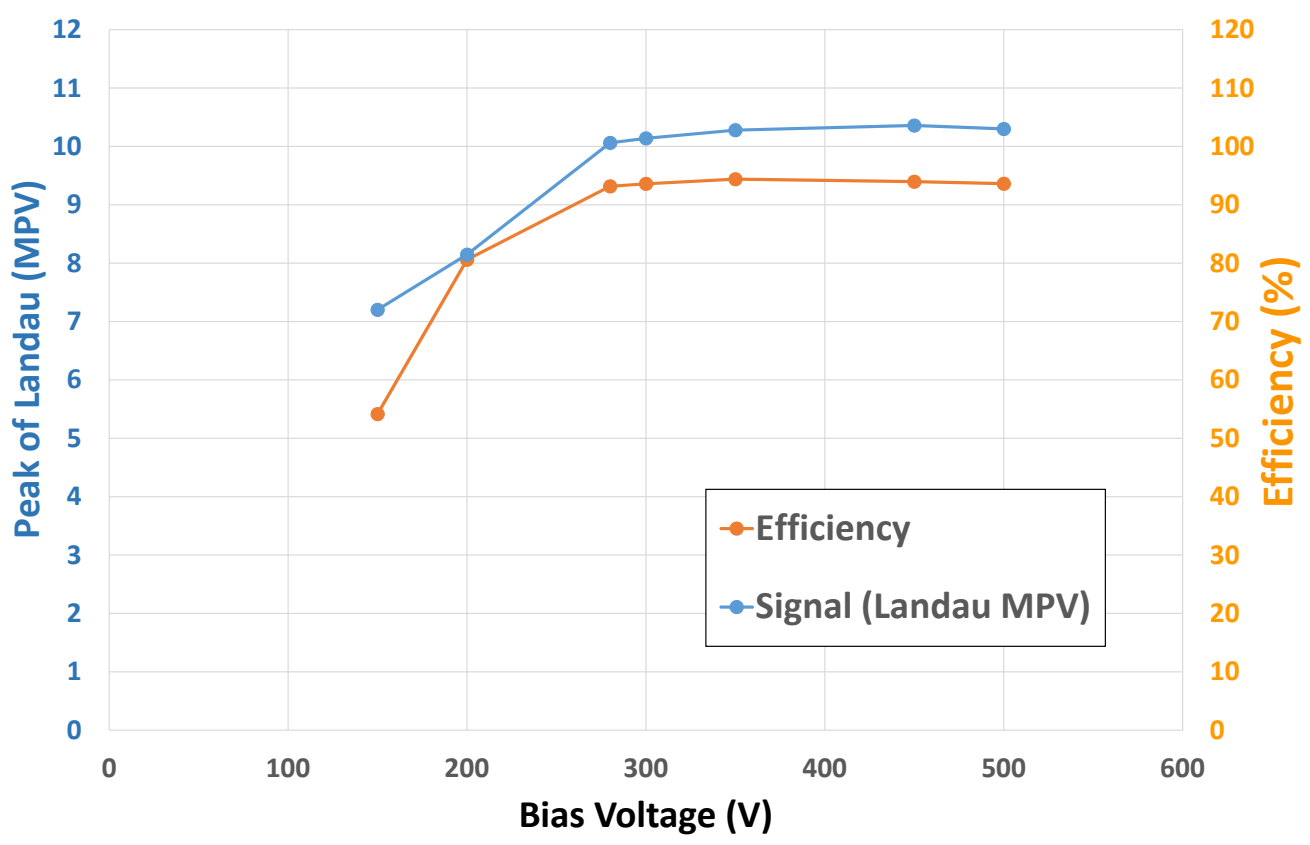

Figure 16: Peak of the Landau fit and efficiency of the Type B UT sensor versus the applied bias voltage. The sensor was irradiated to twice the maximum expected fluence prior to the beam test.

Figure 17 shows the charge collected in each of the four time bins for the data taken at $350 \mathrm{~V}$ bias, for DUT clusters matched to charged tracks. The best time bin is time bin 0 , the top left. As before, we see there is one best time bin, one worst time bin, and two somewhere in between. For the remaining plots, the focus should be on the results for time bin 0 .

Figure 18 shows the Landau fit to the cluster charge distribution with a bias of $350 \mathrm{~V}$. The peak of the Landau is at about 10.3 ADC counts, which corresponds to about $9 \%$ less charge collected by the irradiated Type B sensor compared to the unirradiated Type A sensor. This loss of charge collection is expected due to defects in the crystal lattice, which lead to charge trapping [11].

Figure 19 shows the (left) $\Delta x$ distribution and (right) number of strips in the cluster, for clusters matched within $0.5 \mathrm{~mm}$ of a track (approximately 10 times the strip pitch). The UT sensor bias voltage is $350 \mathrm{~V}$. The vertical red lines show the width of a strip. Almost all hits are within \pm 0.5 of the strip pitch, and most hits are single-strip hits.

Figures 20, 21 and 22 show the average cluster size, average cluster charge and 

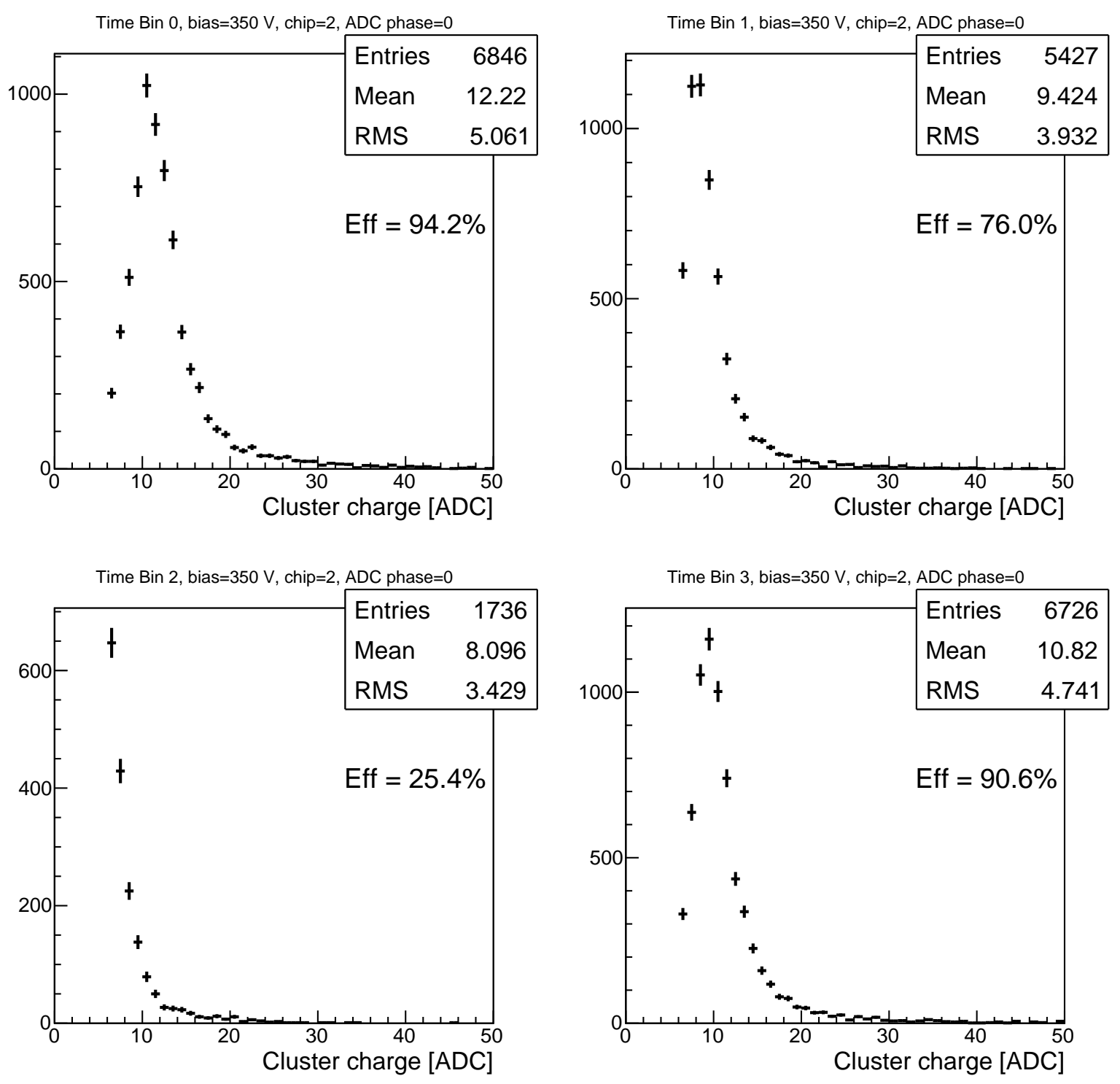

Figure 17: Distributions of collected charge (in ADC counts) for the Type B sensor irradiated to twice the maximum nominal fluence, and biased at $350 \mathrm{~V}$ bias, for the four time bins. The best time bin is the top left (time bin 0).

efficiency versus the interstrip position, respectively, for each of the four time bins. Figure 20(top left) indicates, as expected, that the cluster size is larger when a track hits between two strips compared to directly on a strip. The charge sharing region is also broader than in the Type A sensor. Part of this is due to the narrower pitch, since 0.1 unit in this plot corresponds to about $9.3 \mu \mathrm{m}$, whereas for the Type $\mathrm{A}$ it is about $18.8 \mu \mathrm{m}$. However, some of it is likely due to changes in the temporal and spacial charge collection properties in irradiated sensors. This effect has been seen in previous UT test beam studies [5].

Figure 21 (top left) shows about a 10\% drop in charge collected at the middle between two strips compared to it striking on top of a strip. Figure 22 (top left) shows that when a particle passes through the strip center, the efficiency is about $98 \%$. However, at the 


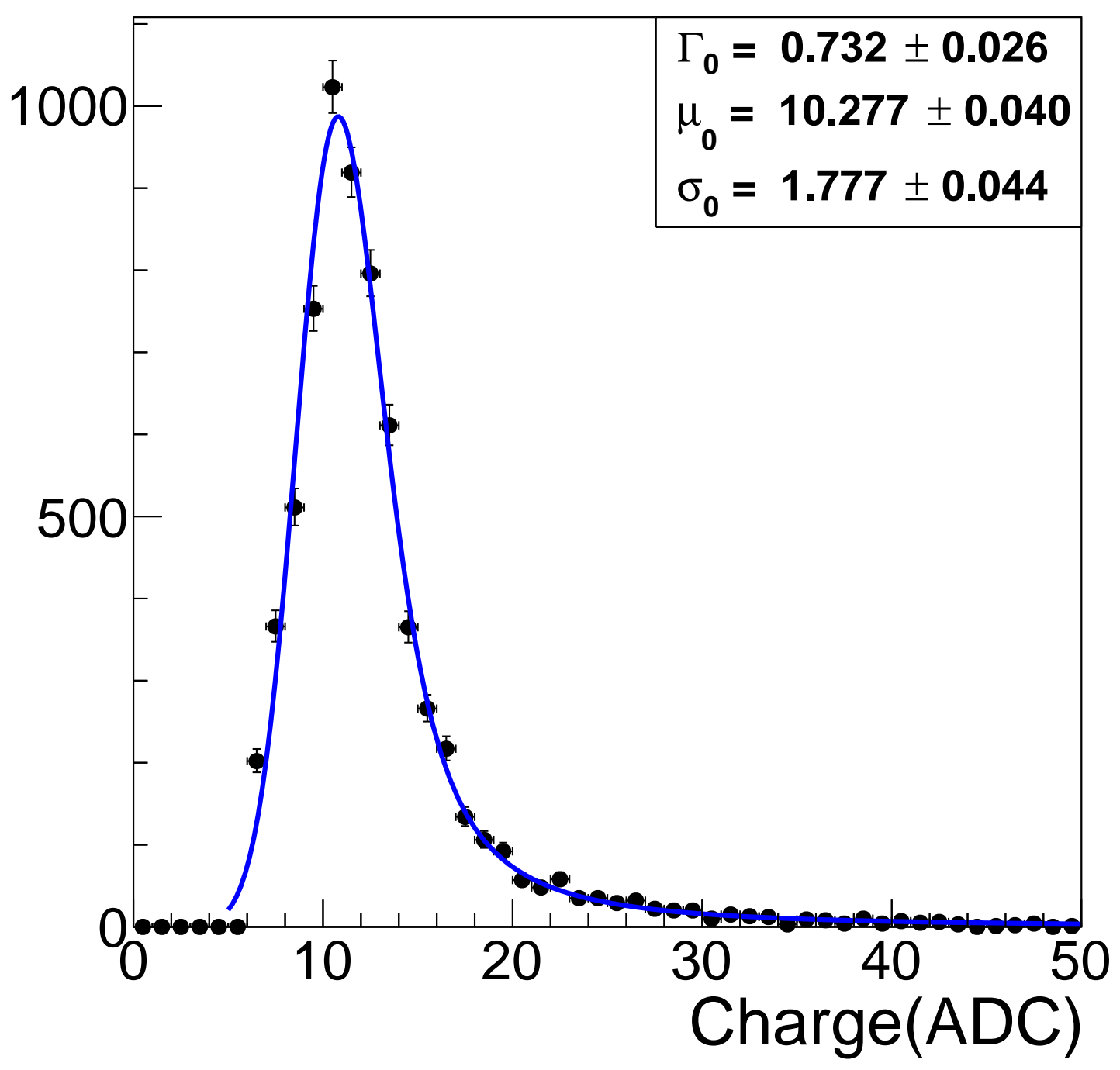

Figure 18: Distributions of collected charge (in ADC counts) for the Type B sensor irradiated to twice the maximum expected fluence, with the bias voltage set at $350 \mathrm{~V}$ bias.

middle between two strips (zero in the plot), the efficiency drops to about $88 \%$. This loss of efficiency occurs because when the particle passes in between two strips, the charge is split and shared between the two adjacent strips. When the charged is shared symmetrically, there is a higher probability that both strips fail the minimum ADC requirement of 6 . If the charge is shared unequally, the probability increases that at least one strip is above threshold. This is the reason for the observed drop in efficiency. We reiterate that a threshold of $\mathrm{ADC} \geq 6$ was only required due to the feature/bug with MiniDAQ1 described previously, which required a very low noise rate. In the final system in LHCb, this will not be the case, and we expect to run at a threshold closer to 3 or at most 4 ADC counts. In that case, even with a peak ADC value of 10, we would expect to be nearly $100 \%$ efficient. 
Bias $=350 \mathrm{~V}$, chip $=2$, ADC phase $=0$

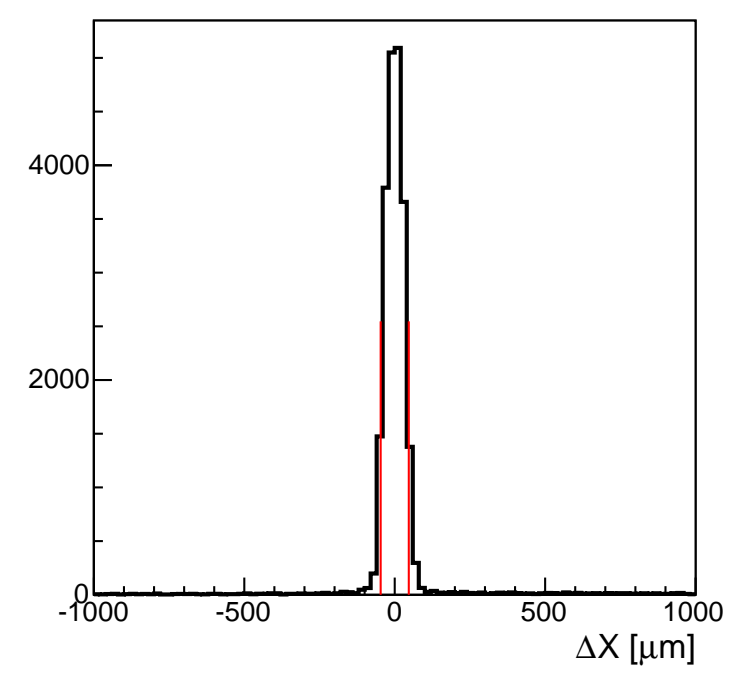

Bias $=350 \mathrm{~V}$, chip $=2$, ADC phase $=0$

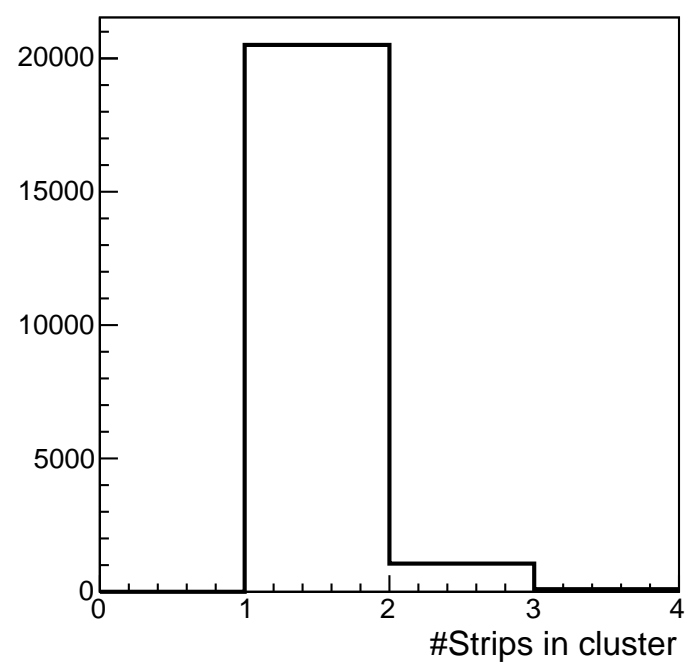

Figure 19: Distribution of (left) $\Delta x=x_{\mathrm{UT}}-x_{\text {track }}$ and (right) number of strips in a cluster, for clusters within $1 \mathrm{~mm}$ of a telescope track for the type B irradiated sensor at $350 \mathrm{~V}$ bias. 

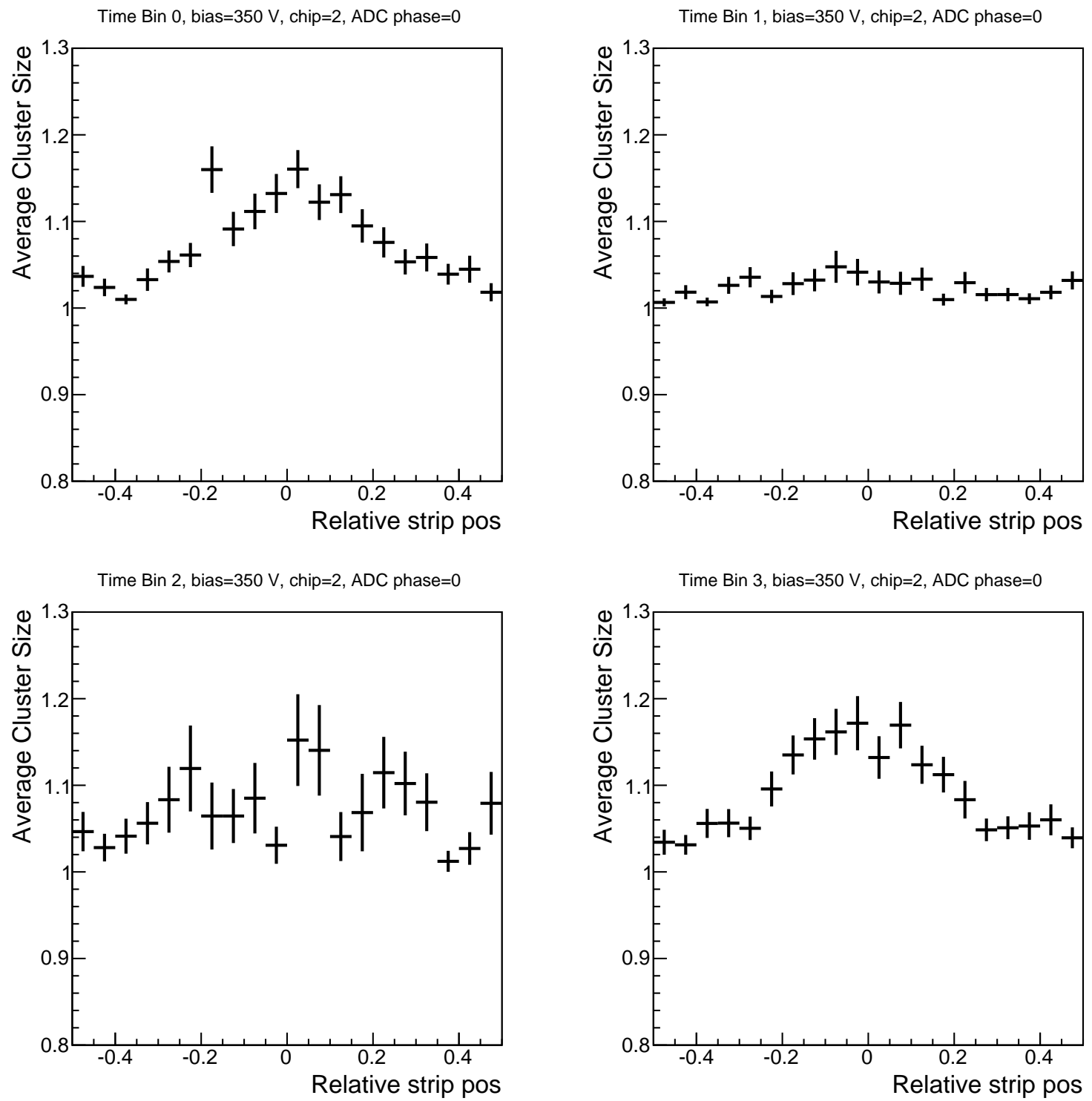

Figure 20: Average cluster size versus the interstrip position for each of the four time bins for the irradiated Type B sensor. The most relevant one is time bin $=0$ (top left). 

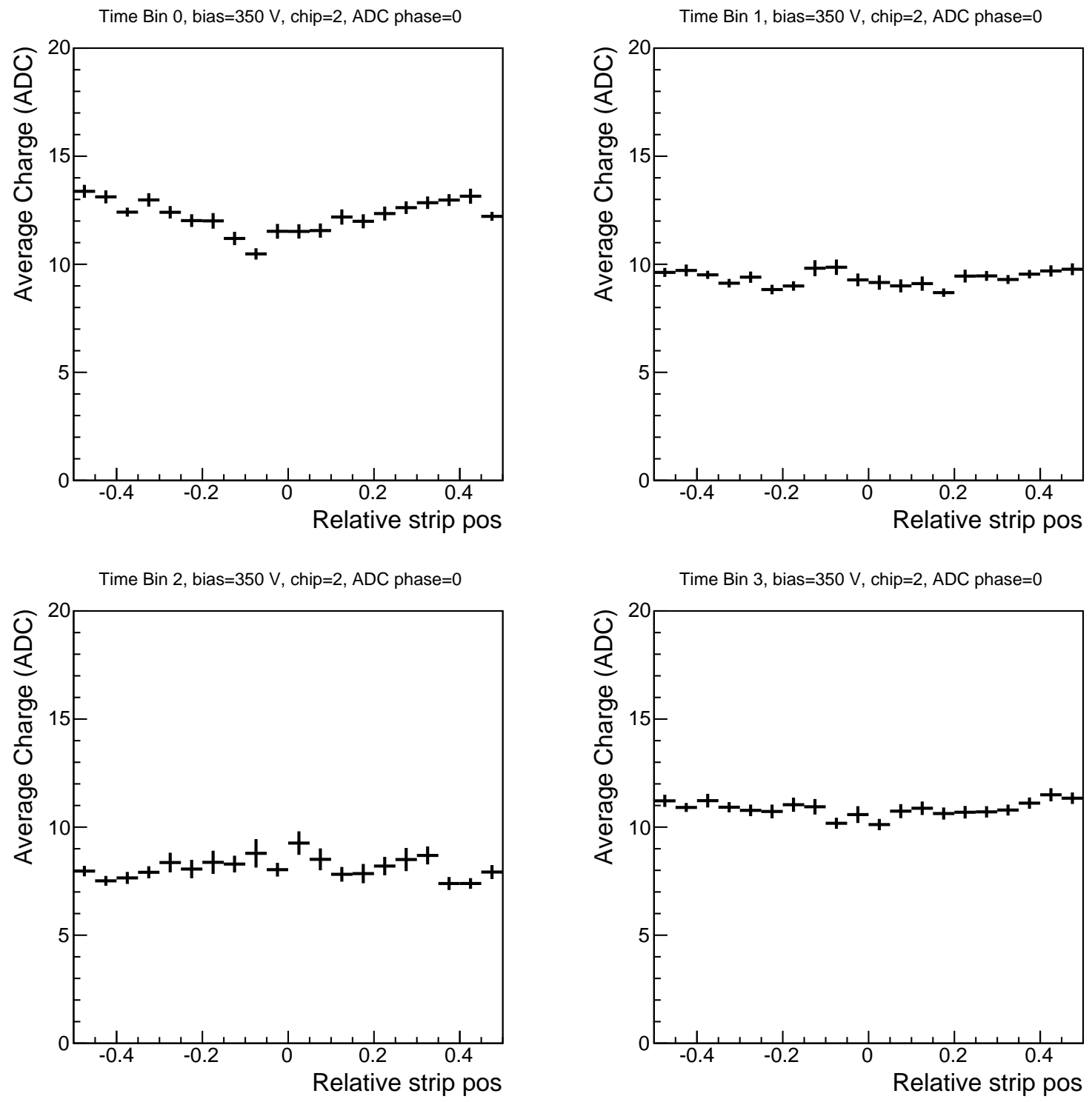

Figure 21: Average cluster charge (in ADC) versus the interstrip position for each of the four time bins for the irradiated Type B sensor. The most relevant one is time bin $=0$ (top left). 

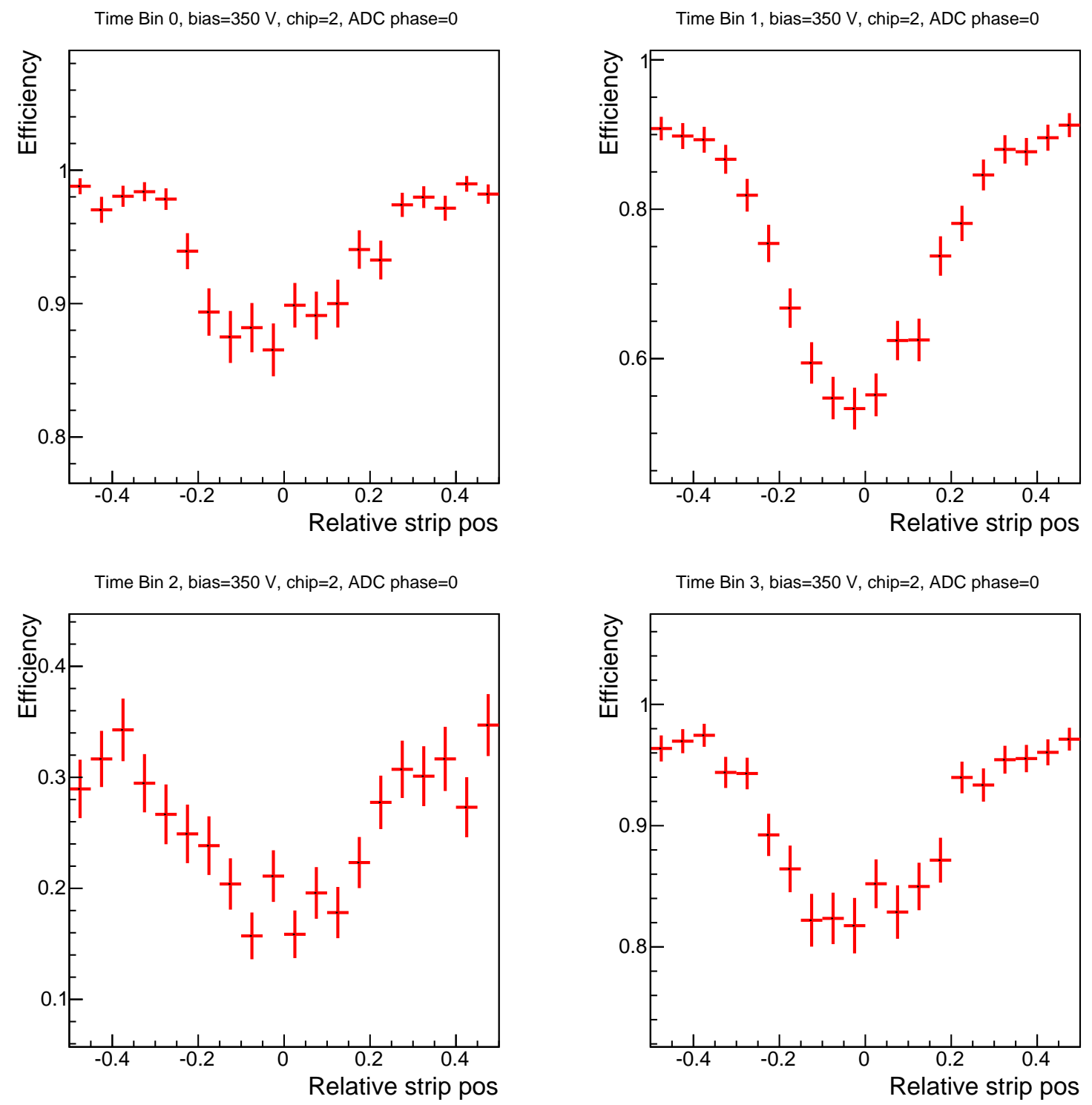

Figure 22: Efficiency versus the interstrip position for each of the four time bins for the irradiated Type B sensor. The most relevant one is time bin $=0$ (top left). 


\section{Summary}

Beam tests of an unirradiated Type A sensor and an irradiated Type B sensor, each connected to SALT 3.0 readout electronics, have been carried out the Fermilab Test Beam Facility in March 2019. This beam test represents the first such test with the final version of the SALT ASIC (v3.0). The tests show that the chip functions properly, and can be used for the full production. The results demonstrate a $\mathrm{S} / \mathrm{N}$ of about 12 for the unirradiated Type A sensor, and a hit efficiency of at least $99.5 \%$. For the Type B sensor, which was irradiated to twice the maximum nominal fluence, as $\mathrm{S} / \mathrm{N}$ of about 11 is achieved, with a efficiency of about $94 \%$. Specific and technical issues with the MiniDAQ1 required that the tests be run with a higher than nominal threshold of about $6 \sigma_{\text {noise }}$. In the final system, we expect to be able to operate at a threshold closer to $3-4 \sigma_{\text {noise }}$, which should keep the hit efficiency well above $99.5 \%$ over the life of the detector.

In the UT, tracks will be required to have at least $3 \mathrm{UT}$ hits. With this definition, we show in Table 1 the efficiency for having 4 UT hits, 3 UT hits, or less than 3 UT hits on a track, as a function of an assumed single-hit efficiency. A single-hit efficiency of at least $99.5 \%$ is the goal for UT, for which the efficiency of finding at least 3 UT hits is $99.99 \%$. Even in the case that the average single-hit efficiency is $98 \%$, we still should have an overall efficiency of $99.77 \%$ for finding at least 3 UT hits for a given track.

Table 1: Percentage of tracks in UT with all 4 UT hits, 3 out of 4 UT hits, or less than 3 hits detected (inefficiency), as a function of the average single-hit efficiency, $<\epsilon_{\text {hit }}>$.

\begin{tabular}{lccc}
\hline \hline$\left\langle\epsilon_{\text {hit }}\right\rangle(\%)$ & $f(4$ hit $)(\%)$ & $f(3$ hit $)(\%)$ & Inefficiency $(\%)$ \\
\hline 99.8 & 99.20 & 0.80 & 0.00 \\
99.5 & 98.01 & 1.97 & 0.01 \\
99.0 & 96.06 & 3.88 & 0.06 \\
98.5 & 94.13 & 5.73 & 0.13 \\
98.0 & 92.24 & 7.53 & 0.23 \\
97.5 & 90.37 & 9.27 & 0.36 \\
97.0 & 88.53 & 10.95 & 0.52 \\
\hline \hline
\end{tabular}

\section{Acknowledgements}

We express our gratitude to the Fermilab accelerator staff and the personnel at the Fermilab Test Beam facility for providing us with the opportunity to perform the measurements presented in this note. Specifically, we thank Lorenzo Uplegger for his support in helping set up and operating the telescope, and for implementing the necessary code to provide tracking information to us. We are also gratful for the organizational and administrative support of Evan Niner and Mandy Rominsky to ensure that we could make optimal use of the beam time upon our arrival. 


\section{References}

[1] LHCb collaboration, LHCb Tracker Upgrade Technical Design Report, CERN-LHCC2014-001, 2014.

[2] M. Firlej et al., A fast, low-power, 6-bit SAR ADC for readout of strip detectors in the LHCb upgrade experiment, Journal of Instrumentation 9 (2014) P07006.

[3] M. Firlej et al., SALT3 chip documentation, See https://twiki.cern.ch/twiki/pub/LHCb/StripAsic/salt_v5_spec.pdf .

[4] See Beetle manual http://www.kip.uni-heidelberg.de/lhcb/Publications/BeetleRefMan_v1_3.pdf.

[5] A. Abba et al., Testbeam studies of pre-prototype silicon strip sensors for the lhcb ut upgrade project, Nuclear Instruments and Methods in Physics Research Section A: Accelerators, Spectrometers, Detectors and Associated Equipment 806 (2016) 244.

[6] A. Abba et al., Study of prototype sensors for the Upstream Tracker Upgrade, LHCbPUB-2016-007, 2016, See https://cds.cern.ch/record/2137551/files/LHCb-PUB-2016007.pdf .

[7] See https://ftbf.fnal.gov/ for addtional information.

[8] P. Durante et al., in MiniDAQ1: A compact data acquisition system for GBT readout over 10G ethernet at LHCb: Volume 1, pp. 332-336, 2018.

[9] K. Wyllie et al., Electronics architecture of the LHCb Upgrade, See https://cds.cern.ch/record/1340939/files/LHCb-PUB-2011-011.pdf .

[10] Particle Data Group, M. Tanabashi et al., Review of particle physics, Phys. Rev. D98 (2018) 030001.

[11] G. Kramberger et al., Modeling of electric field in silicon micro-strip detectors irradiated with neutrons and pions, Journal of Instrumentation 9 (2014) P10016. 